\title{
Extracellular Vesicles: Evolving Factors in Stem Cell Biology
}

\author{
Muhammad Nawaz, ${ }^{1,2}$ Farah Fatima, ${ }^{1,2}$ Krishna C. Vallabhaneni, ${ }^{3}$ Patrice Penfornis, ${ }^{3}$ \\ Hadi Valadi, ${ }^{2}$ Karin Ekström, ${ }^{4,5}$ Sharad Kholia, ${ }^{6}$ Jason D. Whitt, ${ }^{3}$ Joseph D. Fernandes, ${ }^{3}$ \\ Radhika Pochampally, ${ }^{3}$ Jeremy A. Squire, ${ }^{1}$ and Giovanni Camussi ${ }^{7}$ \\ ${ }^{1}$ Department of Pathology and Forensic Medicine, Faculty of Medicine Ribeirao Preto, University of Sao Paulo, Avenue Bandeirantes, \\ 3900 Ribeirao Preto, SP, Brazil \\ ${ }^{2}$ Department of Rheumatology and Inflammation Research, University of Gothenburg, Box 480, 40530 Gothenburg, Sweden \\ ${ }^{3}$ Cancer Institute, University of Mississippi Medical Center, Jackson, MS 39216, USA \\ ${ }^{4}$ BIOMATCELL VINN Excellence Center of Biomaterials and Cell Therapy, 40530 Gothenburg, Sweden \\ ${ }^{5}$ Department of Biomaterials, University of Gothenburg, Box 412, 40530 Gothenburg, Sweden \\ ${ }^{6}$ Cellular and Molecular Immunology Research Centre, School of Human Sciences, London Metropolitan University, \\ London 166-220, UK \\ ${ }^{7}$ Department of Medical Sciences and Molecular Biotechnology Center, University of Torino, Corso Dogliotti 14, 10126 Torino, Italy
}

Correspondence should be addressed to Muhammad Nawaz; nawaz@usp.br and Giovanni Camussi; giovanni.camussi@unito.it

Received 13 May 2015; Revised 9 July 2015; Accepted 16 July 2015

Academic Editor: Alexander Kleger

Copyright (C) 2016 Muhammad Nawaz et al. This is an open access article distributed under the Creative Commons Attribution License, which permits unrestricted use, distribution, and reproduction in any medium, provided the original work is properly cited.

Stem cells are proposed to continuously secrete trophic factors that potentially serve as mediators of autocrine and paracrine activities, associated with reprogramming of the tumor microenvironment, tissue regeneration, and repair. Hitherto, significant efforts have been made to understand the level of underlying paracrine activities influenced by stem cell secreted trophic factors, as little is known about these interactions. Recent findings, however, elucidate this role by reporting the effects of stem cell derived extracellular vesicles (EVs) that mimic the phenotypes of the cells from which they originate. Exchange of genetic information utilizing persistent bidirectional communication mediated by stem cell-EVs could regulate stemness, self-renewal, and differentiation in stem cells and their subpopulations. This review therefore discusses stem cell-EVs as evolving communication factors in stem cell biology, focusing on how they regulate cell fates by inducing persistent and prolonged genetic reprogramming of resident cells in a paracrine fashion. In addition, we address the role of stem cell-secreted vesicles in shaping the tumor microenvironment and immunomodulation and in their ability to stimulate endogenous repair processes during tissue damage. Collectively, these functions ensure an enormous potential for future therapies.

\section{Introduction}

Stem cell technology has recently attracted considerable attention in translational medicine due to the potential that these cells possess in terms of tissue regeneration and repair and as drug delivery tools for which existing therapeutic strategies pose enduring challenges. In recent years, the fields of regenerative and translational medicine have proven to be very attractive owing to the discovery of novel cellular and noncellular therapeutic platforms for tissue repairs and cancer treatments.
This review mainly engages studies carried out on the two major types of stem cell lines: embryonic stem cells (ESCs) and mesenchymal stem cells (MSCs). Nevertheless, several other types of stem cells closely related to their tissue of origin (e.g., adipose stem cells, cancer stem cells) have also been reported.

ESCs are pluripotent cells with the ability to differentiate into cells from any of the three germ layers: mesoderm, endoderm, and ectoderm. They have the capability to selfrenew and proliferate limitlessly, but their application in 
research and therapy has been limited due to ethical concerns on availability and the risk of forming teratomas.

In the last two decades, more attention has been diverted towards MSCs as they are easily obtainable and show therapeutic promise. MSCs are a nonhematopoietic, heterogeneous population of plastic-adherent cells that exhibit a fibroblast-like morphology. They form distinct colonies when seeded at clonal densities, and their heterogeneity is distinguished through morphological differences, rate of proliferation, and their ability to differentiate [1]. According to the current nomenclature, human MSCs can be identified through their positivity for cell surface markers such as CD73, CD90, and CD105 and the lack of expression of hematopoietic markers such as CD11b or CD34, CD45, CD79 or CD19, and HLA-DR [2]. Furthermore, they must have the ability to differentiate into osteoblasts, chondrocytes, and adipocytes in vitro [2]. The biological effects of MSCs depend largely on their ability to secrete trophic factors that stimulate tissueintrinsic progenitor cell phenotypes [3]. These factors include growth factors, miRNAs, and small vesicles that not only potentially affect the differentiation and regenerative abilities of MSCs but also play a critical role in mediating crosstalk to local and distant tissues through which stem cell populations maintain a stable coexistence [4].

Recent evidence shows that stem cells secrete small vesicles into the extracellular milieu, known as extracellular vesicles (EVs). EVs are submicron vesicles, which based on their size, origin, morphology, and mode of release can be categorized into exosomes $(40-200 \mathrm{~nm})$, microvesicles (50$1000 \mathrm{~nm})$, apoptotic bodies $(50-5000 \mathrm{~nm})$, or Golgi vesicles (reviewed in [5]). EVs are secreted by a multitude of cell types into various body fluids [6] and can be isolated via several conventional as well as high throughput technologies [5]. They are known to carry a repertoire of mRNAs, miRNAs, DNA, proteins, and lipids that can be transferred to neighboring cells, modifying their phenotype as well as the microenvironment $[7,8]$. The molecular signatures of EVs are selective to each cell/tissue type, which makes them ideal source for clinical applications [5].

The biogenesis and secretion of EVs from biologically active cells are a stimulus dependent event that is arising as a result of tumor progression or repair processes. A wellstudied process of formation of exosomes is by the fusion of the multivesicular endosome with plasma membrane and release by the process of exocytosis. Conversely, microvesicles are less well characterized in comparison to exosomes and are produced as a result of membrane budding processes and detachment of spherical bodies from discriminatory regions of the plasma membrane (for mechanisms, see [5]). Recently, Golgi vesicles were highlighted, considering them a part of the extracellular vesicle populations as separate entities. Like other vesicles, they may reflect distinct disease states [5], and their role could be hypothesized for underappreciated effectors in stem cell context.

Interestingly, it has been shown recently that MSC exosomes are derived from endocytosed lipid-raft microdomains [9]. Arguably, the knowledge about the mechanisms of biogenesis and origin of EV populations from stem cells could enhance our understanding of the functional relevance and help acquire therapeutic possibilities using stem cell-EVs.

\section{Intercellular Communication and Transfer of Biological Information}

Cells use several means of communication for the exchange of materials and the transfer of information in order to maintain tissue homeostasis, cell development, repair, and survival. Intercellular communication is therefore considered one of the most important regulatory mechanisms for cell growth, differentiation, and tissue remodeling. This intercellular crosstalk may occur through direct receptor-mediated interaction between cells, or through cellular junctions (i.e., cell fusion). Certain molecules, specific transmembrane proteins, and cell adhesion molecules such as integrins, tetraspanins, and cadherins are known to promote receptor-mediated cellular interactions that are critical in the formation of biological patterns as well as determining cell fates $[10,11]$. On the other hand, the direct coupling of the cytoplasm of two cells through gap junctions (GJs) has also been reported to play an essential role in maintaining tissue homeostasis, development, and stem cell differentiation [12, 13].

It has been proposed that normal human adult stem cells do not express gap junctional proteins (i.e., connexins) and do not appear to have gap junctional intercellular communication. However, their differentiated, nonstem cell derivatives do express connexins and therefore utilize GJs for intercellular communication in order to differentiate [14]. Remarkably, bone marrow derived MSCs have been reported to differentiate into cells with a cardiac phenotype in response to signals from neighboring myocytes, as a result of gap junctional intercellular communication [15]. Arguably, the crucial role that these interactions play in the maintenance of tissue integrity in a plethora of different cell types such as endothelial cells, fibroblasts, adipocytes, cardiomyocytes, and neurons is of critical translational importance.

\subsection{Tunneling Nanotubes and Intercellular Communication.} Recently, a novel mechanism for cellular communication involving long distance intercellular connections called tunneling nanotubes (TNTs) has come to light [16]. TNTs are actin-based cytoplasmic extensions that not only facilitate direct communication between distant cells and intercellular trafficking [17] but also are associated with the transfer of biomolecular cargos such as organelles, Golgi vesicles, plasma membrane components, and even pathogens [18-20]. These functions of TNTs have therefore implicated them in the promotion of various physiological (tissue development and regeneration [21]) as well as pathological processes such as mesothelioma [22].

For instance, extensive spontaneous intercellular exchange of cytosolic materials and organelles, between primary human proximal tubular epithelial cells, was recently reported [23]. Interestingly, this exchange was alleviated significantly on inhibiting TNT genesis, therefore implying the importance of TNTs in renal physiology [23]. Surprisingly, the direct transfer of genetic material between tumor and stromal cells has also been identified to be 
mediated through TNTs, exclusive of other forms of cell-cell communication, therefore suggesting a role of TNTs in tissue remodeling as well [24]. A recent study described the nanotubular connections and tubule fragments in multipotent MSCs from human arteries, demonstrating that they interact constitutively through an articulate and dynamic tubule network allowing long-range cell-to-cell communication [25]. This therefore suggests that one mechanism by which MSCs exhibit their protective effects on the heart may be through tubular communication.

\subsection{Cell-Cell Communication through Paracrine Secreted} Factors. Diffusion of autocrine and paracrine signaling molecules enables cells to communicate in the absence of physical contact. The beneficial effects of stem cells are therefore restricted not only to cell-to-cell contact alone, but also to their transient paracrine actions through the release of a combination of trophic factors including cytokines, chemokines, and growth factors [26-28]. These factors have been shown to have various functional properties such as being antiapoptotic, immunomodulatory, and angiogenic, playing a role in cell-mobilization, evoking responses from resident cells in the extracellular environment, and facilitating collateral remodeling [29]. Through receptor-mediated interactions, these paracrine mechanisms potentially facilitate the homing of stem cells to sites of tissue injury, therefore allowing recipient cells to interact with stem cell factors that may influence the regulation of differentiation and antiinflammatory responses [26].

Cells could also communicate through secreted extracellular RNAs, which, once transferred from one cell to another, may modulate the phenotype and function of the recipient cells. Furthermore, the exchange of extracellular RNAs between resident cells and MSCs has been implicated in the modulation of cell fate [30]. These extracellular RNAs are secreted, vesicle-encapsulated or in association with proteins that confer protection from degrading enzymes and contribute towards the microenvironmental modulation of cell fate with other extracellular factors such as the extracellular matrix as well as the local biochemical and mechanical niches [31].

\subsection{Extracellular Vesicles as Novel Means of Communication.} Secreted EVs exhibit novel paracrine mechanisms mediating cell-to-cell communication through receptor-ligand mediated interactions and/or direct fusion with resident cells, resulting in the horizontal transfer of various proteins as well as genetic material (including mRNA $[8,32-34]$ and miRNA [34]). As paracrine factors, EV s have been reported to induce phenotypic changes in recipient cells and also generate a functional link between stem cells and tissues under various physiological and pathological circumstances (reviewed in [33]).

Recently, it has been demonstrated that EVs released by human adipocytes or adipose tissue-explants are involved in a reciprocal proinflammatory loop between adipocytes and macrophages in a paracrine fashion, with the possibility to aggravate insulin resistance locally or systemically [35]. Moreover, EVs released from osteoblasts serve as a mechanism of communication between osteoblasts and osteoclasts through receptor-ligand interactions to facilitate osteoclast formation that represent a novel mechanism of bone remodeling [36].

Although stem cells have been considered safe for available therapeutic strategies, there are still potential risks and complications that may arise such as culture-induced senescence, immune-mediated rejection, genetic instability, loss of functional properties, and consequent malignant transformations. In this perspective, there is a prospect that stem cell-EVs may overcome these limitations and therefore offer novel and safe cell-free therapeutic opportunities. However, their promise as real clinical applications still awaits further investigations yet to be fully realized.

\section{Extracellular Vesicles Mimic Stemlike Phenotypes}

Recent data from various different studies have shown stem cell-EVs to traffic stem cell associated transcription factors such as Nanog, Oct-4, HoxB4, and Rex-1 operating at the level of pluripotent stem cells [37]. On top of that, they have also been shown to express MSC markers such as CD105 [38], prominin-1/CD133 [39-41], and KIT [42]. More direct effectors of the stem cell phenotype such as WNT $[43,44]$, $\beta$-catenin [45], and Hedgehog [46] have also been identified on stem cell-EVs together with several other components that may be considered potential factors in stem cell biology (Table 1). Furthermore, ESC-EVs can be harvested from exponentially growing ESCs, therefore suggesting that the mechanism of EV release from ESCs is inherent [37].

Emerging evidence reveals that secreted vesicles from stem cells could mimic a spectrum of stemlike phenotypes. Ratajczak and coworkers provided the first evidence that EVs released from stem cells might modulate the phenotype of recipient cells [37]. In this study, they reported that EVs released from ESCs sustained the maintenance of hematopoietic stem/progenitor cell stemness and multipotency by delivering specific proteins and mRNA, subsequently upregulating the expression of early pluripotent and hematopoietic stem cell genes [37]. A following study further confirmed this by reporting that mRNA-carrying EVs from endothelial progenitors induced a proangiogenic phenotype in quiescent endothelial cells [47]. Moreover, it was recently revealed that EV-mediated mRNA delivery into marrow cells could introduce tissue-specific changes through the induction of transcription in recipient cells [48], further indicating that mRNAs present in stem cell-EVs are functional and have regulatory effects.

Several studies have revealed that the key biological features of stem cells, such as self-renewal, differentiation, and maturation, could be mimicked by stem cell associated EVs [49-53]. Furthermore, stem cell-EVs have been reported to coordinate physiological self-regenerative and repair processes after tissue injury in a paracrine manner. For instance, EVs released from MSCs (MSC-EVs) activate repair processes by transferring miRNAs [54] and promoting angiogenesis $[55,56]$, therefore suggesting EVs as functional extensions of stem cells. 
TABLE 1: Stem cell signatures expressed and secreted in EVs.

\begin{tabular}{|c|c|c|}
\hline Components & Stem cell type/source & Reference \\
\hline $\begin{array}{l}\text { Nanog, Oct-4, HoxB4 } \\
\text { and Rex-1 }\end{array}$ & ESCs & {$[37]$} \\
\hline CD105 & CSCs, MSCs & {$[38,97]$} \\
\hline Prominin-1/CD133 & HPCs, melanocytes & {$[39,40]$} \\
\hline KIT & Mast/stem cells & [42] \\
\hline WNT & Fibroblasts, DLBCL & {$[43,44,96]$} \\
\hline$\beta$-catenin & HEK & {$[45]$} \\
\hline Stem-cell antigen-1 & MSCs & {$[97]$} \\
\hline TGF- $\beta 1$ & $\begin{array}{l}\text { Fibroblasts, epithelial } \\
\text { cells }\end{array}$ & {$[98]$} \\
\hline $\begin{array}{l}\text { TIA, TIAR, HuR, Staul, } \\
\text { Stau2, RPS29, and Ago } 2\end{array}$ & MSCs & {$[54]$} \\
\hline KGF & MSCs & [99] \\
\hline Oct4 and Sox 2 mRNAs & ESCs & {$[62]$} \\
\hline IGF-1R mRNA & MSCs & {$[100]$} \\
\hline $\begin{array}{l}\text { DEFA3, HBB, ITGA2B, } \\
\text { and ITGB3 mRNAs }\end{array}$ & HPCs & [101] \\
\hline VEGF & MSCs & {$[68]$} \\
\hline $\begin{array}{l}\text { PDGFR- } \beta \text {, TIMP } 1 \text { and } \\
2 \text {, sphingomyelin, lactic } \\
\text { acid, and glutamic acid }\end{array}$ & MSCs & {$[69]$} \\
\hline CD34 & MSCs & {$[102]$} \\
\hline E-cadherin & MSCs & {$[103]$} \\
\hline $\mathrm{Bcl}-2$ & MSCs & {$[104]$} \\
\hline NEP & MSCs & {$[105]$} \\
\hline
\end{tabular}

ESCs: embryonic stem cells, CSCs: cancer stem cells, MSC: mesenchymal stem cells, HPCs: haematopoietic precursor cells, DLBCL: diffuse large Bcell lymphoma, HEK: human embryonic kidney cells, KIT: mast/stem cell growth factor receptor, IGF-1R: growth factor receptor, TGF- $\beta 1$ : transforming growth factor beta 1, KGF: keratinocyte growth factor, ITGA; integrin alpha, VEGF: vascular endothelial growth factor, PDGFR- $\beta$ : platelet-derived growth factor receptor beta, TIMP: tissue inhibitor of metalloproteinase, and NEP: neprilysin.

Interplay between stem cells and EVs reveals that EVs could transmit multipotential traits and represent several features of MSCs, such as multilineage differentiation, selfrenewal, and transcriptional regulation, therefore, inferring their role as evolving factors in stem cell biology.

\section{Stem Cell-EVs Contribute to the Cell-Fate Determination}

There is sufficient evidence to postulate that EVs carry biological messages from parent cells that interact with recipient cells and influence their normal physiology and therefore their overall fate [33]. In the context of stem cell biology, it is likely that the same principle applies (Figure 1), as biological content from stem cell-EVs has been shown to have the capability to influence and define cell fates of future populations of resident cells, potentially producing long lasting and stable transformation in genetic programs $[48,57]$. Furthermore, Quesenberry et al. have also proposed that EV-mediated exchange of genetic information could be an integral element of the continuum model of stem cell biology, in which the differentiation decision of stem cells is conditioned by the cell cycle transit and environmental stimuli [58].

4.1. EV-Mediated miRNA Transport and Cell-Fate Determination. To sustain stemness, a set of transcriptional factors are required to efficiently reprogram and self-renew the pluripotency of recipient cells to enable them to differentiate into various cell types [59]. Indeed, miRNAs are capable of targeting and regulating the expression of stem cell associated transcriptional networks by which they can induce phenotypic transition $[60,61]$. In this context, EVs contribute to such reprogramming and phenotypic switching by transferring miRNAs as the delivery of selective sequestered miRNAs in EVs to recipient cells has been reported [34].

For instance, EVs from bone marrow- (BM-) MSCs shuttle the selected pattern of miRNAs to recipient cells targeting genes involved in multiorgan development, cell survival, and differentiation [54]. Furthermore, ESC-EVs induce dedifferentiation and the expression of pluripotency factors in their target cells by selective transfer of mRNA and miRNA, which may initiate an early differentiation process by targeting early transcriptional factors [62]. It has also been reported that telocytes (TCs) secrete EVs loaded with miRNAs that could modulate stem cell fates through continuous posttranscriptional regulatory signals acting back and forth between TCs and stem cells [63]. Moreover, Let-7 miRNA family expressed from human embryonic MSCs has been shown to affect its downstream target hepatic nuclear factor 4 alpha (HNF4A), indicating the possible role of stem cells in differentiation processes through EV-mediated transfer of miRNAs [64]. In addition, several other miRNAs have been identified to potentially contribute to regulatory mechanisms in stem cell biology (Table 2). These reports therefore signify that stem cells may exhibit their biological effects through EV-mediated shuttle of miRNAs for the biological effects mediated by stem cells.

\section{Stem Cell-EVs and Tumor Progression}

The role of stem cells in tumor progression has been well documented [65]; however, the mechanisms by which cancer stem cell-derived EVs initiate and promote tumor progression are still uncertain. Recent evidence explains the interplay between stem progenitors and their secreted vesicles in tumor progression. For example, it has been reported that EVs positive for the stem cell marker prominin-1 participate in Wnt signaling and mediate prometastatic activity in melanoma cells [40]. In addition, EVs from mast cells express and shuttle KIT protein (mast/stem cell growth factor receptor), which in turn promotes tumor growth in recipient lung adenocarcinoma cells by activating the KIT-SCF signaling pathway [42]. EVs from BM-MSCs have also been shown to express higher levels of oncogenic proteins, cytokines, and adhesion molecules that could be transferred to multiple myeloma cells and modulate tumor growth in vivo [66]. 


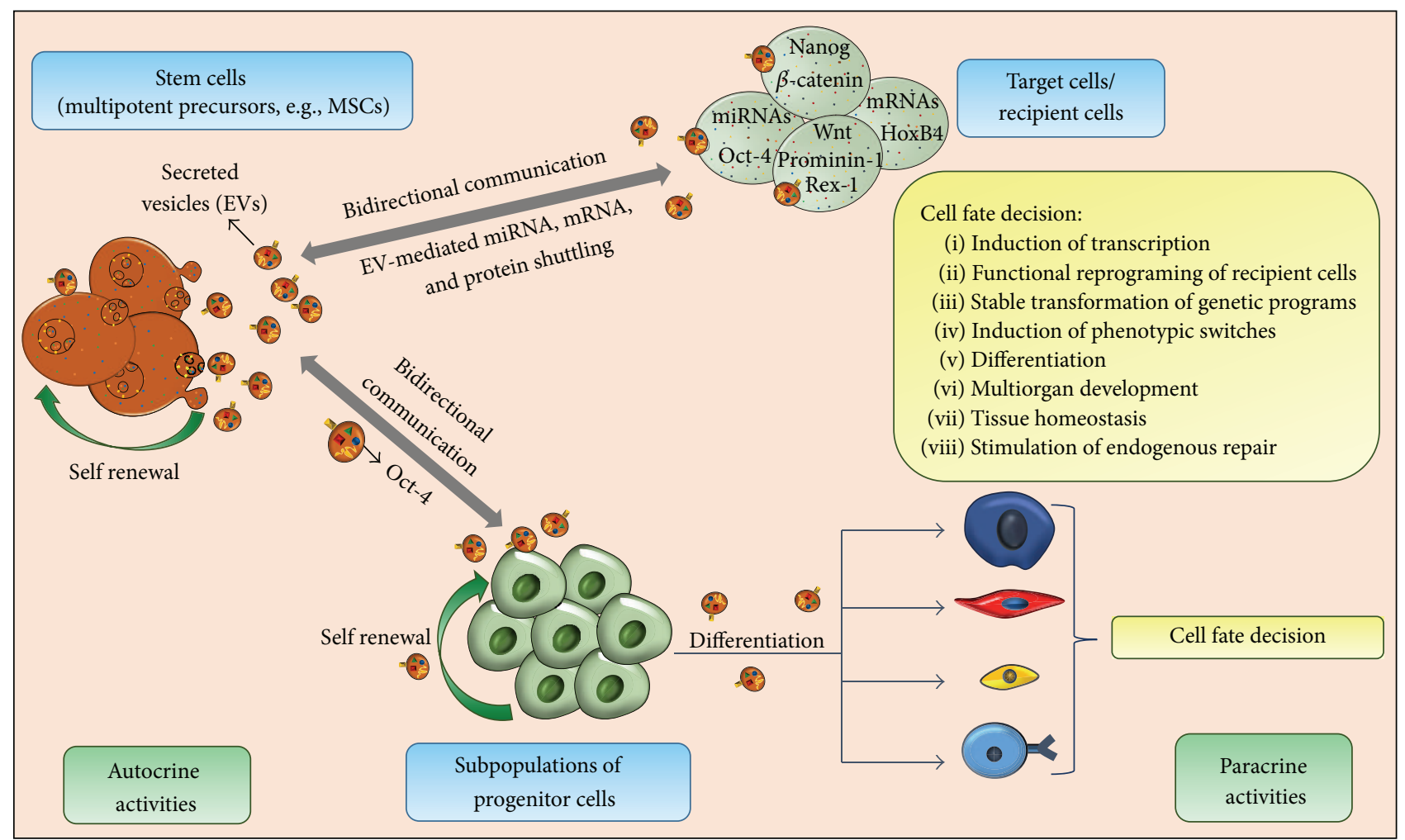

Figure 1: The role played by stem cell-derived EVs in the determination of cell fate. Stem cells use EVs to transfer miRNAs and stem cell effectors in recipient cells, which target the regulatory networks and induce persistent genetic transformation and phenotypic switching of resident cells towards cell-fate determination.

TABLE 2: Selectively enriched regulatory miRNAs from stem cellderived EVs.

\begin{tabular}{llc}
\hline miRNAs & $\begin{array}{l}\text { Stem cell } \\
\text { type/source }\end{array}$ & Reference \\
\hline $\begin{array}{l}\text { miR-199b, miR-218, miR-148a, } \\
\text { miR-135b, and miR-221 }\end{array}$ & MSCs & {$[106]$} \\
miR-223, miR-564, miR-451, and & MSCs & \\
miR-142-3p & ESCs & {$[54]$} \\
miRNAs of 290 cluster & MSCs & {$[62]$} \\
let-7 miRNA family & MSCs & {$[64]$} \\
miR-133b & MSCs & {$[107]$} \\
miR-15a & MSCs & {$[66]$} \\
miR148a, miR532-5p, miR378, & MSCs & {$[67]$} \\
miRNA-21, 34a & MSCs & {$[69]$} \\
miR-23b & MSCs & {$[73]$} \\
miR-16 & Preadipocytes & {$[108]$} \\
miR-140 & MSCs & {$[109]$} \\
miR-22 & MSCs & {$[70,110]$} \\
miR-221 & CPCs & {$[111]$} \\
miR-210, miR-132, and & ESCs & {$[112]$} \\
miR-146a-3p & & \\
miR-294 & & {$[74$} \\
\hline
\end{tabular}

Recently, Eirin et al. reported that adipose MSC (AT-MSC) derived EVs enriched with distinct mRNAs and miRNAs regulate various physiological processes such as angiogenesis, adipogenesis, apoptosis, and proteolysis in recipient cells [67]. Furthermore, EVs from BM-MSCs have been reported to not only transport tumor regulatory miRNAs, antiapoptotic proteins, and metabolites but also enhance the expression of vascular endothelial growth factor (VEGF) through the activation of extracellular signal-regulated kinase $1 / 2(E R K 1 / 2)$ pathway to promote growth of various tumors $[68,69]$. In addition, gastric cancer derived MSC-EVs have been shown to deliver deregulated miRNAs to human gastric cancer cells and promote their proliferation and migration [70].

A subset of tumor-initiating cells expressing the MSC marker CD105 in human renal cell carcinoma release EVs that induce angiogenic phenotype in endothelial cells and promote the formation of a premetastatic niche [38, 71]. Furthermore, renal cell carcinoma-derived EVs induce persistent phenotypical changes in MSCs accompanied with enhanced expression of genes associated with cell migration, matrix remodeling, angiogenesis, and tumor growth [71].

To the contrary, various reports also suggest the antitumorigenic effects of EVs from multiple stem cell types. For instance, Bruno et al. have reported that BM-MSCs exert inhibitory effects on tumor growth through the release of EVs [72]. Furthermore, BM-MSC derived EV-mediated transfer 
of miRNAs from the bone marrow could promote dormancy in metastatic breast cancer cells [73]. In addition, various studies have reported the antiproliferative effects of MSCEVs through various mechanisms, for instance, miRNAmediated VEGF suppression in breast cancer cells [74] and the downregulation of phosphorylation of Akt kinase in bladder tumor cells [75]. Similarly, a study by Fonsato et al. shows that EV-mediated delivery of selected miRNAs from adult human liver stem cells (HLSC) exhibits inhibitory effects on hepatoma growth [76]. On the basis of this information and on various other reports, MSC derived EVs could therefore exert either anti- or protumorigenic effects depending on the tumor type and stage of development [77].

Glioma-associated stem cells (GASCs) produce substantial amounts of EVs that express and mediate glioma-stem cell characteristics [78-80]. For instance, a recent study reported that GASCs exhibit stem cell feature and anchorageindependent growth and are capable of sustaining malignant properties of both glioma cells and glioma-stem cells, mainly through the release of EVs [78]. Furthermore, the cargo (DNA, miRNA, transcripts, and proteins) from GASC derived EVs may influence lineage specific dynamics of the stem cell compartments [81].

MSC-EVs could also be engineered to play a potential role in cancer therapeutics as delivery vehicles. For instance, Munoz et al. successfully reported the delivery of functional anti-miR-9 to glioblastoma (GBM) cells through MSC-EVs, therefore increasing their level of chemosensitivity substantially [79]. In addition, EV-dependent phenotypes of stem cells such as neurosphere growth and endothelial tube formation were attenuated by loading miR-1 into GBM-EVs that were exposed to the glioblastoma microhabitat [80]. Furthermore, a recent study by Katakowski et al. observed that intratumor injection of miR-146 expressing MSC-EVs significantly inhibited glioma xenograft growth [82]. Likewise, Pascucci et al. also reported the potential ability of MSCs to package/incorporate and deliver active drugs such as paclitaxel through EVs to inhibit tumor progression [83]. These studies therefore indicate the potential capability and utility of stem cell-EVs as drug delivery mechanisms for cancer therapy.

5.1. Ways by Which Stem Cell-EVs Influence the Tumor Microenvironment. The principle properties of cancer stem cells (CSCs) are maintained by niches which are anatomically distinct regions within the tumor microenvironment [84]. The premetastatic niche plays a role in dormancy, relapse, and the development of metastasis. It has been hypothesized that EVs from CSCs may behave as metastasomes, helping the implementation of secondary lesions by transmission of the metastatic phenotype via EV-borne tumor RNA signatures to the target organ [85]. Since the construction of a premetastatic niche is an essential early step required for initiated cells to survive and evolve [86], it could be speculated that stem cells may contribute to the construction of premetastatic niches, at least in part, by secreting EVs. This could be supported through the observation of Grange et al., whereby interactions between endothelial cells and CSCs induced phenotypical changes in MSCs and promoted the formation of lung premetastatic niches through the release of EVs [38].

5.1.1. Fibroblastic Differentiation and Stroma. Tumor cells have the ability to efficiently "educate" MSCs to induce changes in the local microenvironment through the release of EVs. For instance, tumor cell-derived EVs have been observed to propagate the construction of the tumor stroma through fibroblastic differentiation of MSCs [87]. Similarly, EVs from ovarian cancer cells have also been reported to induce adipose stromal cells (ASCs) to acquire the characteristics of tumor-supporting myofibroblasts [88]. A similar phenotypic switch was also noted in prostate cancer (PC) EVs whereby they influenced the differentiation of MSCs into proangiogenic and proinvasive myofibroblasts that was associated with disease promotion [89]. In another study, EVs from PC cells induced phenotypic transformation in PC patient ASCs to form prostate-like neoplastic lesions [90]. On top of that, tumor derived EVs can initiate phenotypic differentiation of MSCs into cancer-associated fibroblasts (CAFs) through signaling pathways [91]. These activated CAFs influence tumor progression through the secretion of metalloproteinases-rich EVs that promote cell motility and activate RhoA and Notch signaling in cancer cells [92]. Moreover, these EVs also play a role in chemoresistance and tumor relapse by promoting clonogenicity and growth of CSCs which are inherently resistant to cell death [93].

5.1.2. Crosstalk among Stromal Elements. EV-mediated dynamic crosstalk within the stroma could relocalize the oncogenic factors that can generate a tumor-initiating niche. This can be speculated from the fact that carcinogenesis involves the relocalization of CAFs to the tumor site therefore sustaining metastasis [94]. The general involvement of EVs in intercellular communication suggests that they may also have a role in information exchange within stem cell hierarchies, whereby cancer stemlike cells may transmit signals to their stroma via EVs. Undeniably, it is well known that ESC-EVs transfer mRNAs, miRNAs, and proteins to recipient cells and therefore are important mediators of crosstalk within stem cell niches [95].

Stromal cell-derived EVs can interact with cancer cells and exchange oncogenic signatures present in tumorassociated stroma. For example, intercellular communication mediated by fibroblast-secreted EVs promotes cancer cell motility via autocrine Wnt-planar cell polarity signaling pathway to drive invasive activities [43]. Furthermore, the Wnt3a has been shown to be exported via EVs to neighboring cells which in turn modulates the population equilibrium in the tumor towards progression [96]. Such features are expected to be established very early during tumorigenesis; however, the prolonged intercellular communication could eventually be sustained and ultimately aggravate tumor growth. It could also be anticipated that the tumor microenvironment may host nontumorigenic multipotent stem cells that could likewise support the biological activity of tumor-initiating cells through the release of EVs. 
5.1.3. Endothelial Cell Growth and Angiogenesis. Angiogenesis is one of the underlying hallmarks of a developing tumor microenvironment. It is activated by proangiogenic factors such as VEGF thereby promoting tumor growth, invasion, and metastases. In response to hypoxia (a hallmark of developing tumors), MSCs increase EV production, which in turn promotes angiogenesis [113] and endothelial cell growth in vitro [114]. This proangiogenic property of stem cell-secreted EVs is reported to be linked with the activation of the ERK signaling pathway therefore increasing the expression of VEGF in tumor cells [68]. Furthermore, ATMSC derived EVs have also been implicated to influence ATMSC induced angiogenesis. Interestingly, the platelet-derived growth factor (PDGF) was identified to elicit this effect by stimulating AT-MSCs to release EVs with an enhanced proangiogenic potential [115]. In addition, AT-MSC derived EVs have been shown to interact with endothelial cells as well, thereby stimulating proangiogenic activity in the tumor microenvironment, possibly through the exchange of angiogenic growth factors [116].

EV-assisted targeting of key regulatory signaling pathways in the tumor microenvironment such as Wnt, Hedgehog, and angiogenic pathways (i.e., VEGF) could inhibit tumor growth. A recent study by Gernapudi et al. showed that a critical signaling axis, miR-140/SOX2/SOX9, which regulates differentiation, stemness, and migration in the tumor microenvironment, could be targeted to obstruct tumor progression [108]. Likewise, EV-assisted targeting of the VEGF pathway in the tumor microenvironment could exert antiangiogenic effects and inhibit tumor growth [74]. However, monitoring a complex stromal network in a dynamic tumor microenvironment has been and still remains technically challenging.

In summary, EVs secreted from cancer cells educate MSCs to undergo neoplastic transformation into tumorassociated fibroblasts in local stroma. In addition, EVmediated dynamic interactions amongst stromal elements and the concomitant recruitment of oncogenic CAFs, growth factors, immune molecules, and several other related mechanisms shape a tumor niche capable of development and growth (Figure 2).

\section{Stem Cell-EVs: Emerging Factors in Immunomodulation and Immunotherapy}

There is increasing evidence implicating EVs to play a critical role in immune regulatory mechanisms comprising of both immunoactive and suppressive activities [117119]. Conversely, studies elucidating the role of EVs from immunologically active stem cells are seldom reported in the scientific literature, possibly because their role as potential factors in immunomodulation is still novel. MSCs have been shown to secrete immunologically active EVs, which induce higher expression of anti-inflammatory transcripts such as interleukin 10 (IL10) and transforming growth factor $\beta 1$ (TGF $\beta 1$ ) and attenuated levels of proinflammatory transcripts such as IL1B, IL6, tumor necrosis factor $\alpha$ (TNF $\alpha)$, and IL12P40 [120]. Furthermore, infusion of MSC-EVs significantly enhanced the survival and delayed the rejection of allogenic skin graft in mice with a corresponding increase in regulatory $\mathrm{T}$ cells (Tregs) therefore indicating some role of EVs in immunomodulation [120].

Islet derived MSCs have been reported to release immunostimulatory EVs that could activate autoreactive B and $\mathrm{T}$ cells in nonobese diabetic (NOD) mice. Furthermore, immunization with EVs also promotes the expansion of transferred diabetogenic $\mathrm{T}$ cells and accelerates the effector $\mathrm{T}$ cell-mediated destruction of islets suggesting that stem cell-EVs act as autoantigen carriers with the potential to trigger autoimmune responses [97]. On the other hand, human adipose MSC derived EVs have been reported to exert inhibitory effects on the differentiation and activation of $\mathrm{T}$ cells followed by reduced T cell proliferation and IFN- $\gamma$ release, therefore suggesting MSC-EVs to play a potential therapeutic role in the treatment of inflammation-related diseases [121]. Furthermore, EVs from immune cells can also have an effect on MSC cells. For instance, Ekström et al. showed that monocyte-derived EVs can induce increased gene expression of osteogenic markers in human MSCs, indicating that monocyte-derived EVs play a role in bone healing [122].

EVs from acute myeloid leukemia- (AML-) blasts that are positive for the hematopoietic progenitor cell antigen CD34 have been reported to be biologically active. Upon coincubation with natural killer (NK) cells, these AMLblast EVs efficiently downregulated the expression of surface NKG2D (an activator of NK cells) indicating a possible function in mediating immunosuppression in AML [123]. In addition, immunocaptured blast-derived EVs could also serve as biomarkers for AML during the course of therapy as a measure of disease progression or response to therapy.

Using a model of human-into-mouse xenogeneic graftversus-host disease ( $\mathrm{x}-\mathrm{GVHD}), \mathrm{BMSCs}$ were shown to play a role in immunomodulation, mediated by human CD4+ Th1 cells [124]. Notably, recipients treated with BMSCs had elevated levels of exosomes expressing CD73 in the serum that promoted the accumulation of adenosine ex vivo. Furthermore, on inhibiting the adenosine A2A receptor, the immunomodulatory property of BMSCs was abrogated, therefore implying a possible role of BMSC-EVs in an adenosine based immunosuppressive mechanism mediated in a paracrine fashion [124]. Recently, MSC-EVs were tested in a human patient to treat GVHD, whereby they were shown to be well tolerated [125]. Interestingly, the EV based treatment resulted in a reduced proinflammatory cytokine response to the patient's peripheral blood mononucleated cells (PBMCs) in vitro and the clinical GVHD symptoms improved significantly shortly after the start of the EV therapy [125].

Although initial findings ascertain that EVs could be a promising therapeutic source for the treatment of immuneinflammatory diseases [126, 127], translating this technology into EV-mediated stem cell therapy to achieve promising outcomes with minimized toxic effects and safety risks still needs to be explored. 


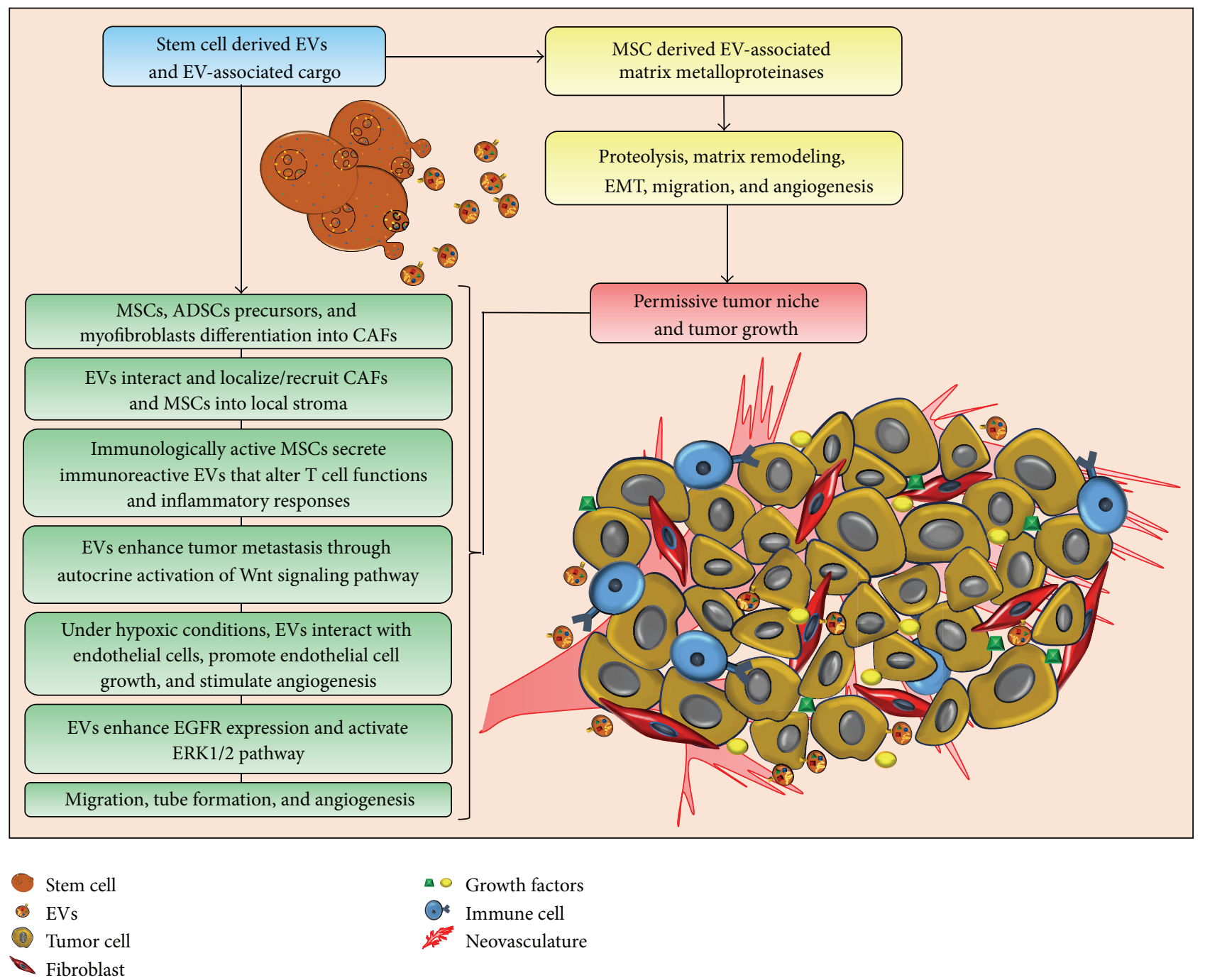

FIGURE 2: Contribution of stem cell-derived EVs in the construction of the tumor microenvironment. Stem cell-derived EVs influence the presence of cancer-associated fibroblasts (CAFs), inflammatory immune cells, metalloproteinases, angiogenic growth factors, and regulatory RNAs, which shape the tumor microenvironment. Extracellular matrix (ECM) remodeling, endothelial cell growth, cell migration, and angiogenesis generate a permissive tumor niche.

\section{Stem Cell-EVs and Tissue Repair}

The therapeutic applications of stem cells for the treatment of various injuries and diseases have received considerable attention in recent years. However, investigations into the possibility of utilizing stem cell-EVs in regenerative medicine are still in their early stages. Recently, it has come to light that stem cells, particularly MSCs, use EVs for tissue repair and regeneration through the transfer of transcription factors, anti-inflammatory factors, and growth factors to target cells in a paracrine fashion. In this section, we discuss the regenerative abilities of MSC-EVs to recover vascular functions, repair injuries, and restore tissue homeostasis.

7.1. Stem Cell-EVs: Allies in the Fight against Cardiovascular Diseases. Stem cells have been suggested as an ideal novel therapeutic approach against cardiovascular diseases.
However, an important feature of this approach is that EVs subsequently released from stem cells are a newly discovered source of cardiovascular protection. Improved heart function and vessel formation have been attributed to EVs released from stem cells, largely due to their anti-inflammatory and antiapoptotic effects (discussed below). Moreover, their effects can equally be extended towards neovascularization and cardiac regeneration.

Stem cell-EVs participate in the suppression of inflammatory responses in order to enhance cardiac functions. For instance, MSC-EVs mediate cytoprotective actions in a model of pulmonary hypertension by suppressing hyperproliferative pathways such as the signal transducer and activator of transcription 3 (STAT3) pathway as well as upregulating miRNAs that are downregulated during pulmonary hypertension [128]. Moreover, MSC-EVs moderate inflammatory reactions and rapidly ameliorate myocardial 
functions during ischemia/reperfusion injury, possibly by restoring bioenergetics, activating PI3K/Akt pathway, and triggering prosurvival signaling [129].

Cardiomyocyte progenitor cells (CPCs) have been shown to stimulate the migration of endothelial cells as well as promoting neovascularization that can potentially improve heart function [130]. Recently, EVs secreted by human CPCs have also been implicated with similar cardioenhancing functions mainly through inhibiting apoptosis, promoting tube formation, and initiating angiogenesis in cardiomyocytic cells [111]. Furthermore, MSC derived EVs also protect cardiac tissue from ischemic injury primarily by enhancing angiogenesis, blood flow recovery, and reducing infarct size [131]. After myocardial infarctions, they are linked with promoting recovery of cardiac functions via the Akt signaling pathway [132].

Ischemic preconditioning can potentiate the protective effects of MSCs during ischemic cardiomyocytes (CMs) injury. This is mainly achieved through the secretion of EVs that deliver miRNAs with antiapoptotic effects [109]. Furthermore, EVs released by the heart after ischemic preconditioning also contribute towards cardioprotection [133]. Interestingly, heat shock proteins have been shown to improve stem cell survival in an ischemic environment. This therefore promotes a prosurvival phenotype in CMs through heat shock factor-1 (HSF-1) and miR-34a interaction that is found to be mediated by EVs [134]. Other than proteins, MSCs are also capable of transferring antiapoptotic miRNAs to CMs via EVs that transcriptionally repress the expression of apoptotic genes, activate cell survival signaling pathway, and ensure cardioprotection $[110,135]$. Such delivery of miRNAs could potentiate antiapoptotic effect of MSCs by inhibiting PUMA (p53-upregulated modulator of apoptosis) to promote cardiomyocyte survival [110].

Injured epithelial cells produce TGF- $\beta 1$-containing EVs with defined genetic information able to activate fibroblasts, which initiate tissue regenerative responses and fibrosis [98]. Cardiac fibrosis is antagonized by stem cell-secreted EVs, exhibiting a substantial protection against myocardial infarction [109]. Furthermore, MSC-EVs have a replacement or cytoprotective effect, thus demonstrating potential therapeutic use in stem cell transplantation for the treatment of pulmonary fibrosis [136]. Notably, ESC-derived EVs can also effectively augment cardiac function in infarcted hearts through enhanced neovascularization, cardiomyocyte survival, and reduced fibrosis after infarction. The underlying basis for this beneficial effect was linked to the delivery of ESC specific miR-294 to CPCs promoting increased survival, cell cycle progression, and proliferation [112]. In addition, CD34+ stem cell-EVs also mediate the transfer of angiogenic factors to the surrounding cells, which may be beneficial in achieving functional recovery after ischemic injury [102]. Therefore, stem cell-EVs from various different tissue locations with their above mentioned healing properties have the potential to act as significant therapeutic agents in the fight against cardiovascular diseases.

7.1.1. Protective and Therapeutic Role against Kidney Injury. MSCs have been implicated in protecting against renal damage through their paracrine effects. By use of a genetic fate-mapping technique, it has been shown that the recovery from acute kidney injury (AKI) depends on the intrinsic regenerative ability of tubular epithelial cells that undergo mesenchymal dedifferentiation and reentry into cell cycle [137]. Experiments performed by Bi et al. show that MSC conditioned medium has the ability to mimic the effects of MSC cells therefore indicating a paracrine form of action [138].

Recent evidence has also demonstrated that MSC-EVs could confer the paracrine actions of their parent cells and therefore contribute towards renal protection as well. For instance, Bruno et al. observed that human MSC-EVs induce recovery in a murine model of AKI in a manner comparable to that of the cell of origin [139]. Furthermore, evidence of mRNAs carried by EVs being translated into proteins has also come to light in both in vitro and in vivo conditions [139]. For example, Tomasoni et al. reported that MSC-EVs mediated the transfer of human IGF-1R mRNA to murine proximal tubular cells, which was subsequently followed by the enhancement of cell sensitivity to the regenerative actions of IGF-1 [100]. EVs from human umbilical cord-derived MSCs (hUC-MSCs) have also been demonstrated to trigger the ERK $1 / 2$ pathway thereby inducing proliferation of tubular cells and protection against cisplatin-induced apoptosis [140].

EVs from human adult MSCs exhibit protective properties against AKI, largely by inhibiting apoptosis and stimulating proliferation of tubular epithelial cells [141]. This was further confirmed by Bruno et al., who showed, in a cisplatininduced lethal model of AKI, that MSC-EVs enhanced the survival of mice through the upregulation of antiapoptotic genes and downregulation of proapoptotic genes [142]. This increased expression was attributed partly to EV-mediated miRNA transfer and in part due to the EV-triggered miRNA transcription [143]. Moreover, miRNA-depleted EVs released by Drosha knockdown MSCs failed to protect the kidney from acute injury [144], indicating that miRNA content of EVs is essential for their biological activity.

Using a mouse remnant kidney model, it was shown that BMSC-EVs could have protective effects against renal injury [145]. Furthermore, endothelial progenitor cell-derived EVs exert a protective effect against mesangial cell injury during Thyl.1 glomerulonephritis [146], and HLSC-derived EVs have the potential to influence renal function and morphology, in a manner comparable to the cells of origin [147]. Administration of MSC-EVs might also remarkably protect mice against renal failure, probably by transferring selective patterns of miRNAs from MSC-EVs to target cells and protection against EMT for restoration [148]. Hence, these studies concur on the therapeutic ability of stem cell-EVs and their potential to be applied as treatment for renal diseases in the future.

7.1.2. Recovering Lung and Liver Injuries. EVs from lung cells have been shown to induce the expression of mRNAs coding for lung specific proteins such as Clara cell protein and aquaporin-5 and A-D surfactants in bone marrow cells [149]. Furthermore, this phenomenon is enhanced significantly during lung injury [149]. 
Acute and chronic lung injuries could in theory be recovered through the paracrine actions of MSC-EVs which confer a stem cell-like phenotype in injured cells for the activation of self-regenerative programs [99]. Moreover, ischemic preconditioning can also potentiate the protective effects of MSCs against endotoxin-induced acute lung injury through the secretion of EVs [150].

HLSC-EVs may also have a role in accelerating the morphological and functional recovery of injured cells during liver damage, mainly through horizontal transfer of specific mRNA subsets to recipient cells [151]. Recently, it has been reported that transplantation of EVs released from hUCMSCs could reduce the surface fibrous capsules, soften their textures, and alleviate hepatic inflammation in fibrotic liver [103]. Moreover, it was observed that human liver cells undergo typical EMT after induction with recombinant human TGF- $\beta 1$, whereas MSC-EV treatment reverses the expression of EMT-associated markers [103], indicating that MSC-EVs could prevent liver fibrosis and ameliorate hepatocyte protection through EMT inhibition. Furthermore, MSC-EVs appear to promote hepatic regeneration following drug-induced liver injury, mainly through the activation of proliferative and regenerative responses [152]. This therefore demonstrates the ability of EVs to act as functional messengers of stem cells.

7.1.3. Stem Cell-EVs and Neuroprotection. There are several studies that have recently come to light that associate MSCEVs with a neuroprotective role along with stem cells. For instance, EV-mediated transfer of miRNAs from MSCs promotes neural plasticity and functional recovery of neurons and astrocytes after treatment for stroke in rats [107]. Furthermore, EV-mediated delivery of miRNAs enhances the expression of glutamate transporters, suggesting an efficient route of therapeutic miRNA delivery to the brain. A recent study by Raisi et al. reported that EVs produced from antiinflammatory MSCs enhanced sciatic nerve regeneration in rats which could be potentially applied in peripheral nerve cell therapy [153]. In addition, EVs from embryonic cerebrospinal fluid have been reported to carry evolutionarily conserved proteins and miRNAs, which promote neural stem cell proliferation [154].

The protective effects of MSC-EVs were recently observed against glutamate-induced excitotoxicity in rat pheochromocytoma via a PI3K/Akt dependent pathway [104]. Investigation of a possible underlying mechanism for this protection revealed that MSC-EVs were responsible for downregulating Bax expression accompanied by reduced cleavage of caspase3, upregulation of $\mathrm{Bcl}-2$ expression, and Akt phosphorylation in glutamate-treated cells, therefore providing a prosurvival fate for affected cells [104]. Transplantation of MSC-EVs could therefore be a potential strategy to treat neuronal diseases involving excitotoxicity.

MSC-EVs may also have effects on neurovascular plasticity during traumatic brain injury by inducing endogenous angiogenesis and neurogenesis and reducing neuronal inflammation $[155,156]$. Furthermore, a study by Katsuda et al. has reported the role of human AT-MSCs derived EVs as a novel therapeutic approach for Alzheimer's disease (AD) as they were found to be loaded with enzymatically active neprilysin (NEP) that potentially contributes to amyloid$\beta$ clearance in the brain [105]. These observations and others therefore support the potential of stem cell-EVs as a means of cell-free therapy against cerebral injuries and neurological diseases.

In summary, stem cells induce regenerative programs in injured tissues of different organs through EV-mediated transfer of anti-inflammatory miRNAs and growth factors. These, in turn, ameliorate the damage (Figure 3 ) by activating different signaling pathways, which have an antiapoptotic and angiogenic effect therefore significantly restoring the bioenergetics, improving blood flow recovery, and inducing stemlike phenotypes in injured organs.

The ability of EVs to mediate bidirectional intercellular communication suggests that they could not only send information from stem cells to injured cells but also communicate from injured cells back to stem cells. In this regard, the future clinical contributions of EVs could therefore be (1) translocation of regenerative signatures at injured sites to trigger repair process, (2) transmission of signals related to tissue injury towards stem cells, to generate more progeny for maintaining stem cell equilibrium and continuous supplies to injured sites, and (3) EVs from injured tissues that could also stimulate stem cells to acquire features of the injured cells.

\section{Concluding Remarks and Future Perspective}

Stem cell-EVs provide a new interpretation of stem cell plasticity that is still a hotly debated topic [157]. EV-mediated bidirectional exchange of information between progenitor cells and tissue differentiated cells leads to phenotypic changes that provide evidence of previously uncertain cell plasticity. This could be beneficial to clinical implications of $\mathrm{EVs}$ for future regenerative medicine.

Elucidating the contribution of stem cell-EVs in premetastatic niche formation and biological characteristics of the cancerous stromal elements may offer novel targeting opportunities and have prognostic and predictive values. However, monitoring the target complex tumor microenvironment remains a technical challenge.

Moreover, the immunomodulatory features of MSC-EVs could be extended in the treatment of immune diseases, reducing potential safety risks originated from conventional immunotherapy such as susceptibility to infection, immune dysfunction, autoimmune responses, and/or increased risk of developing cancer. Conceivably, EVs from immunoreactive MSCs could be novel therapeutic tools against inflammationassociated diseases [125].

Despite the improvements of surgical procedures in organ transplantation and cell-based therapies in the last decade, current methods present potential complications (i.e., toxicity and organ rejection), which remains dismal for improving long-term survival and reducing mortality. It is therefore necessary to look for alternative and more reliable procedures, which could potentially minimize associated risk factors. In this regard, EV based cell-free therapy could be a preferred option as it would improve patients outcome considerably, by reducing the complications associated with 


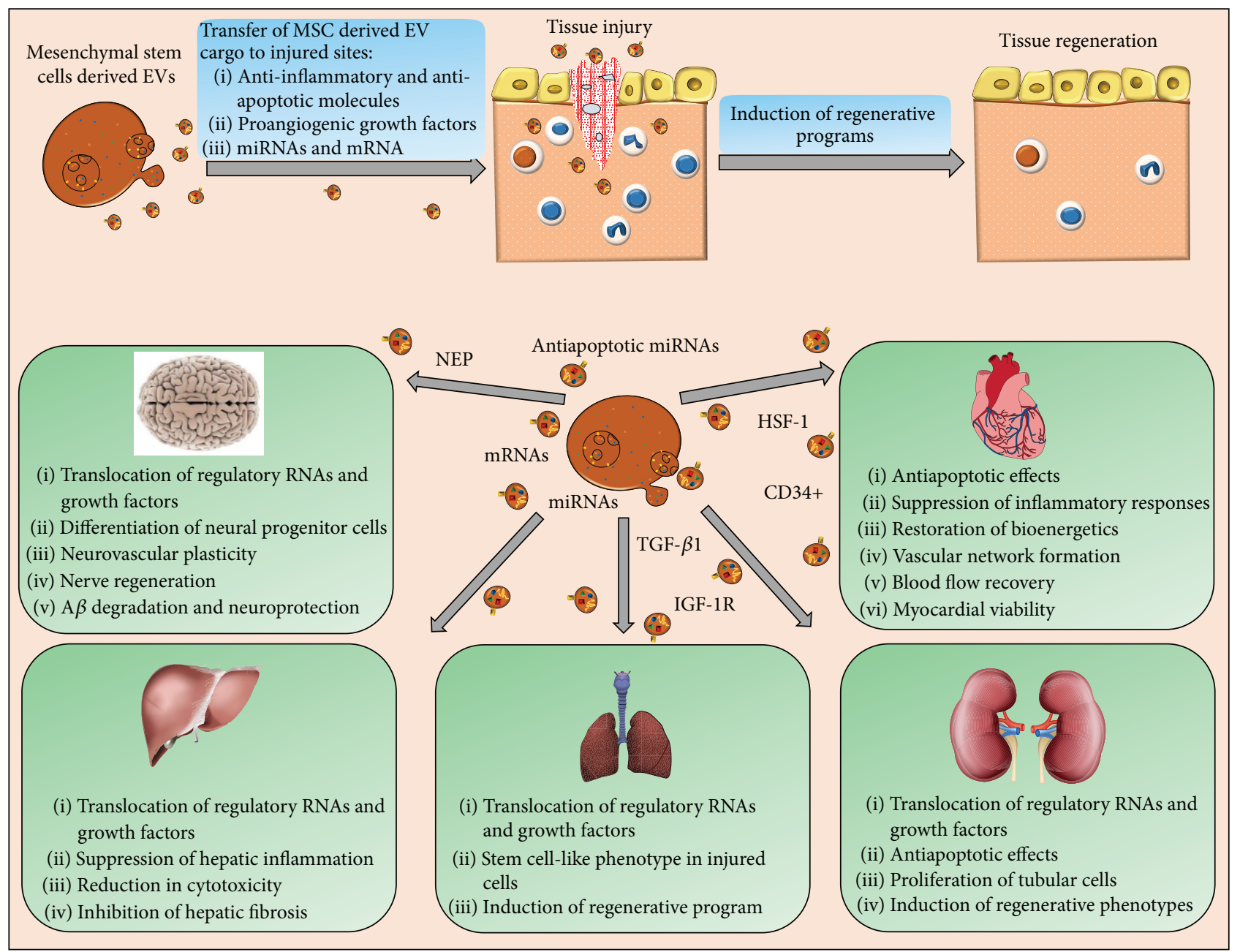

FIGURE 3: Schematic representation of the regenerative effects of stem cell-derived EVs. MSCs use EVs to ameliorate tissue damage through translocating growth factors, anti-inflammatory, antiapoptotic, and proangiogenic molecules, to sites of injury where they induce and regulate regenerative phenotypes.

cell-based treatments. For instance, ESC-EVs provide a novel cell-free system that uses the immense regenerative power of ES cells while avoiding the risks associated with direct ES or ES-derived cell transplantation and risk of teratomas [112]. Furthermore, EVs have the ability to extend the therapeutic effects and functions of stem cells in both a local and systemic environment. Hence, the administration of stem cell-derived EVs would reduce potential safety risks associated with cellular therapy and/or transplantation surgery.

However, further studies are needed to define whether EVs permanently or transiently change the "genetic fingerprint" of an injured target organ and the consequences of it. Preliminary studies on acute kidney injury indicate a restoration of a persistently normal renal molecular pattern after EV treatment [144]. However, the effect may be dose dependent and increasing doses of EVs may produce unwanted effects as we observed in preliminary experiments with adiposederived MSCs.

As MSCs have the potential to differentiate into other types of viable replicating cells, replacing their transplantation with administration of MSC-EVs (which mimic similar functions as MSCs) would reduce this possibility and maintain the therapeutic benefits at the same time. However, the mobilization of EVs and homing to specific tissues are still a major concern. Given that EVs express specific membrane receptors and proteins specific to certain cell types [158], it may be possible to identify a potential mechanism to direct EVs as well as stem cells to a particular tissue. Receptor-ligand mediated interactions and homing could also facilitate the cellular uptake of EVs and their associated cargo by recipient injured cells. Furthermore, as a reparatory response, the mobilization of EVs to injured zones could be a result of dynamic interactions among stromal elements. It is quite possible that, in response to an injury or transplant, the enhanced release of EVs could create a gradient that may educate them to mobilize to the site of injury.

Since EVs are more suitable for monitored manufacturing processes, they could be engineered to express and deliver regenerative signatures to target sites. However, a potential 
challenge is the lack of standardization of the existing technologies to isolate and characterize EVs for their effective therapeutic utility. Therefore, a combination of different high throughput methods could overcome the potential limitations related to EV detection and characterization [5]. Particularly, the technologies for the isolation and characterization of stem cell-EVs must be improved and optimized [159], ensuring the purity of EVs that is critical for developing cell-free therapeutic strategies using stem cell-EVs in the future.

\section{Conflict of Interests}

Authors of this paper declare that there is no potential conflict of interests.

\section{Authors' Contribution}

All authors contributed in intellectual discussions and critical review of the paper. Muhammad Nawaz and Giovanni Camussi arranged data and revised the paper for submission.

\section{Acknowledgments}

This work was supported by funding from Associazione Italiana per la Ricerca sul Cancro (AIRC) IG2012 no. 12890 to G. Camussi. M. Nawaz, F. Fatima, and J. Squire acknowledge FAPESP, CAPES, and CNPq in Brazil. K. Ekström acknowledge The Swedish Research Council (VR 521-20142660) and the BIOMATCELL VINN Excellence Center of Biomaterials and Cell Therapy. The authors searched for original articles in PubMed containing the search terms: "exosomes", "microvesicles", "extracellular vesicles", "mesenchymal stromal cells", "stem cells", and "progenitor cells". All papers identified were English-language, full-text articles. Papers were prioritized on the basis of relevance and the authors apologize to authors of manuscripts that might have been missed.

\section{References}

[1] A. J. Friedenstein, K. V. Petrakova, A. I. Kurolesova, and G. P. Frolova, "Heterotopic of bone marrow. Analysis of precursor cells for osteogenic and hematopoietic tissues," Transplantation, vol. 6, no. 2, pp. 230-247, 1968.

[2] M. Dominici, K. Le Blanc, I. Mueller et al., "Minimal criteria for defining multipotent mesenchymal stromal cells. The International Society for Cellular Therapy position statement," Cytotherapy, vol. 8, no. 4, pp. 315-317, 2006.

[3] A. I. Caplan and J. E. Dennis, "Mesenchymal stem cells as trophic mediators," Journal of Cellular Biochemistry, vol. 98, no. 5, pp. 1076-1084, 2006.

[4] K. X. Wang, L. Xu, Y. Rui et al., "The effects of secretion factors from umbilical cord derived mesenchymal stem cells on osteogenic differentiation of mesenchymal stem cells," PLoS ONE, vol. 10, no. 3, Article ID e0120593, 2015.

[5] M. Nawaz, G. Camussi, H. Valadi et al., "The emerging role of extracellular vesicles as biomarkers for urogenital cancers," Nature Reviews Urology, vol. 11, no. 12, pp. 688-701, 2014.
[6] C. Lässer, V. Seyed Alikhani, K. Ekström et al., "Human saliva, plasma and breast milk exosomes contain RNA: uptake by macrophages," Journal of Translational Medicine, vol. 9, article 9, 2011.

[7] G. Camussi, M.-C. Deregibus, S. Bruno, C. Grange, V. Fonsato, and C. Tetta, "Exosome/microvesicle-mediated epigenetic reprogramming of cells," American Journal of Cancer Research, vol. 1, no. 1, pp. 98-110, 2011.

[8] M. Nawaz, F. Fatima, B. R. Zanetti et al., "Microvesicles in gliomas and medulloblastomas: an overview," Journal of Cancer Therapy, vol. 05, no. 02, pp. 182-191, 2014.

[9] S. S. Tan, Y. Yin, T. Lee et al., "Therapeutic MSC exosomes are derived from lipid raft microdomains in the plasma membrane," Journal of Extracellular Vesicles, vol. 2, Article ID 22614, 2013.

[10] R. O. Hynes, "Integrins: versatility, modulation, and signaling in cell adhesion," Cell, vol. 69, no. 1, pp. 11-25, 1992.

[11] E. C. Lai, "Notch signaling: control of cell communication and cell fate," Development, vol. 131, no. 5, pp. 965-973, 2004.

[12] B. Constantin and L. Cronier, "Involvement of gap junctional communication in myogenesis," International Review of Cytology, vol. 196, pp. 1-65, 2000.

[13] P. C. Schiller, G. D’Ippolito, R. Brambilla, B. A. Roos, and G. A. Howard, "Inhibition of gap-junctional communication induces the trans-differentiation of osteoblasts to an adipocytic phenotype in vitro," Journal of Biological Chemistry, vol. 276, no. 17, pp. 14133-14138, 2001.

[14] J. E. Trosko, "The role of stem cells and gap junctional intercellular communication in carcinogenesis," Journal of Biochemistry and Molecular Biology, vol. 36, no. 1, pp. 43-48, 2003.

[15] M. Xu, M. Wani, Y.-S. Dai et al., "Differentiation of bone marrow stromal cells into the cardiac phenotype requires intercellular communication with myocytes," Circulation, vol. 110, no. 17, pp. 2658-2665, 2004.

[16] A. Rustom, R. Saffrich, I. Markovic, P. Walther, and H.H. Gerdes, "Nanotubular highways for intercellular organelle transport," Science, vol. 303, no. 5660, pp. 1007-1010, 2004.

[17] L. Marzo, K. Gousset, and C. Zurzolo, "Multifaceted roles of tunneling nanotubes in intercellular communication," Frontiers in Physiology, vol. 3, article 72, 2012.

[18] H.-H. Gerdes, N. V. Bukoreshtliev, and J. F. V. Barroso, "Tunneling nanotubes: a new route for the exchange of components between animal cells," FEBS Letters, vol. 581, no. 11, pp. 21942201, 2007.

[19] S. Abounit and C. Zurzolo, "Wiring through tunneling nanotubes-from electrical signals to organelle transfer," Journal of Cell Science, vol. 125, no. 5, pp. 1089-1098, 2012.

[20] E. Lou, S. Fujisawa, A. Morozov et al., "Tunneling nanotubes provide a unique conduit for intercellular transfer of cellular contents in human malignant pleural mesothelioma," PLoS ONE, vol. 7, no. 3, Article ID e33093, 2012.

[21] H.-H. Gerdes, A. Rustom, and X. Wang, "Tunneling nanotubes, an emerging intercellular communication route in development," Mechanisms of Development, vol. 130, no. 6-8, pp. 381387, 2013.

[22] J. W. Ady, S. Desir, V. Thayanithy et al., "Intercellular communication in malignant pleural mesothelioma: properties of tunneling nanotubes," Frontiers in Physiology, vol. 5, article 400, 2014.

[23] S. Domhan, L. Ma, A. Tai et al., "Intercellular communication by exchange of cytoplasmic material via tunneling nano-tube like structures in primary human renal epithelial cells," PLoS ONE, vol. 6, no. 6, Article ID e21283, 2011. 
[24] V. Thayanithy, E. L. Dickson, C. Steer, S. Subramanian, and E. Lou, "Tumor-stromal cross talk: direct cell-to-cell transfer of oncogenic microRNAs via tunneling nanotubes," Translational Research, vol. 164, no. 5, pp. 359-365, 2014.

[25] S. Valente, R. Rossi, L. Resta, and G. Pasquinelli, "Exploring the human mesenchymal stem cell tubule communication network through electron microscopy," Ultrastructural Pathology, vol. 39, no. 2, pp. 88-94, 2015.

[26] T. J. Burdon, A. Paul, N. Noiseux, S. Prakash, and D. Shum-Tim, "Bone marrow stem cell derived paracrine factors for regenerative medicine: current perspectives and therapeutic potential," Bone Marrow Research, vol. 2011, Article ID 207326, 14 pages, 2011.

[27] X. Sun, J. Su, J. Bao et al., "Cytokine combination therapy prediction for bone remodeling in tissue engineering based on the intracellular signaling pathway," Biomaterials, vol. 33, no. 33, pp. 8265-8276, 2012.

[28] K. Tsuji and S. Kitamura, "Trophic factors from tissue stem cells for renal regeneration," Stem Cells International, vol. 2015, Article ID 537204, 7 pages, 2015.

[29] T. Kinnaird, E. S. Burnett, M. Shou et al., "Local delivery of marrow-derived stromal cells augments collateral perfusion through paracrine mechanisms," Circulation, vol. 109, no. 12, pp. 1543-1549, 2004.

[30] L. Guo, R. C. H. Zhao, and Y. Wu, "The role of microRNAs in self-renewal and differentiation of mesenchymal stem cells," Experimental Hematology, vol. 39, no. 6, pp. 608-616, 2011.

[31] D. E. Discher, D. J. Mooney, and P. W. Zandstra, "Growth factors, matrices, and forces combine and control stem cells," Science, vol. 324, no. 5935, pp. 1673-1677, 2009.

[32] J. Ratajczak, M. Wysoczynski, F. Hayek, A. JanowskaWieczorek, and M. Z. Ratajczak, "Membrane-derived microvesicles: important and underappreciated mediators of cell-tocell communication," Leukemia, vol. 20, no. 9, pp. 1487-1495, 2006.

[33] G. Camussi, M. C. Deregibus, S. Bruno, V. Cantaluppi, and L. Biancone, "Exosomes/microvesicles as a mechanism of cell-tocell communication," Kidney International, vol. 78, no. 9, pp. 838-848, 2010.

[34] H. Valadi, K. Ekström, A. Bossios, M. Sjöstrand, J. J. Lee, and J. O. Lötvall, "Exosome-mediated transfer of mRNAs and microRNAs is a novel mechanism of genetic exchange between cells," Nature Cell Biology, vol. 9, no. 6, pp. 654-659, 2007.

[35] M. E. G. Kranendonk, F. L. J. Visseren, B. W. M. Van Balkom et al., "Human adipocyte extracellular vesicles in reciprocal signaling between adipocytes and macrophages," Obesity, vol. 22, no. 5, pp. 1296-1308, 2014.

[36] L. Deng, Y. Wang, Y. Peng et al., "Osteoblast-derived microvesicles: a novel mechanism for communication between osteoblasts and osteoclasts," Bone, vol. 79, pp. 37-42, 2015.

[37] J. Ratajczak, K. Miekus, M. Kucia et al., "Embryonic stem cellderived microvesicles reprogram hematopoietic progenitors: evidence for horizontal transfer of mRNA and protein delivery," Leukemia, vol. 20, no. 5, pp. 847-856, 2006.

[38] C. Grange, M. Tapparo, F. Collino et al., "Microvesicles released from human renal cancer stem cells stimulate angiogenesis and formation of lung premetastatic niche," Cancer Research, vol. 71, no. 15, pp. 5346-5356, 2011.

[39] N. Bauer, M. Wilsch-Bräuninger, J. Karbanová et al., "Haematopoietic stem cell differentiation promotes the release of prominin-1/CD133-containing membrane vesicles-a role of the endocytic-exocytic pathway," EMBO Molecular Medicine, vol. 3, no. 7, pp. 398-409, 2011.

[40] G. Rappa, J. Mercapide, F. Anzanello et al., "Wnt interaction and extracellular release of prominin-1/CD133 in human malignant melanoma cells," Experimental Cell Research, vol. 319, no. 6, pp. 810-819, 2013.

[41] H. B. Huttner, P. Janich, M. Köhrmann et al., “The stem cell marker prominin-1/CD133 on membrane particles in human cerebrospinal fluid offers novel approaches for studying central nervous system disease," Stem Cells, vol. 26, no. 3, pp. 698-705, 2008.

[42] H. Xiao, C. Lässer, G. Shelke et al., "Mast cell exosomes promote lung adenocarcinoma cell proliferation-role of KIT-stem cell factor signaling," Cell Communication and Signaling, vol. 12, article 64, 2014.

[43] V. Luga, L. Zhang, A. M. Viloria-Petit et al., "Exosomes mediate stromal mobilization of autocrine Wnt-PCP signaling in breast cancer cell migration," Cell, vol. 151, no. 7, pp. 1542-1556, 2012.

[44] J. C. Gross, V. Chaudhary, K. Bartscherer, and M. Boutros, "Active Wnt proteins are secreted on exosomes," Nature Cell Biology, vol. 14, no. 10, pp. 1036-1045, 2012.

[45] A. Chairoungdua, D. L. Smith, P. Pochard, M. Hull, and M. J. Caplan, "Exosome release of $\beta$-catenin: a novel mechanism that antagonizes Wnt signaling," Journal of Cell Biology, vol. 190, no. 6, pp. 1079-1091, 2010.

[46] A. C. Gradilla, E. González, I. Seijo et al., "Exosomes as Hedgehog carriers in cytoneme-mediated transport and secretion," Nature Communications, vol. 5, article 5649, 2014.

[47] M. C. Deregibus, V. Cantaluppi, R. Calogero et al., "Endothelial progenitor cell-derived microvesicles activate an angiogenic program in endothelial cells by a horizontal transfer of mRNA," Blood, vol. 110, no. 7, pp. 2440-2448, 2007.

[48] J. M. Aliotta, M. Pereira, K. W. Johnson et al., "Microvesicle entry into marrow cells mediates tissue-specific changes in mRNA by direct delivery of mRNA and induction of transcription," Experimental Hematology, vol. 38, no. 3, pp. 233-245, 2010.

[49] R. Nair, L. Santos, S. Awasthi et al., "Extracellular vesicles derived from preosteoblasts influence embryonic stem cell differentiation," Stem Cells and Development, vol. 23, no. 14, pp. 1625-1635, 2014.

[50] A. Forterre, A. Jalabert, K. Chikh et al., "Myotube-derived exosomal miRNAs downregulate Sirtuinl in myoblasts during muscle cell differentiation," Cell Cycle, vol. 13, no. 1, pp. 78-89, 2014.

[51] D. S. Mistry, Y. Chen, and G. L. Sen, "Progenitor function in self-renewing human epidermis is maintained by the exosome," Cell Stem Cell, vol. 11, no. 1, pp. 127-135, 2012.

[52] J. P. Webber, L. K. Spary, A. J. Sanders et al., "Differentiation of tumour-promoting stromal myofibroblasts by cancer exosomes," Oncogene, vol. 34, no. 3, pp. 290-302, 2015.

[53] S. C. McIver, Y.-A. Kang, A. W. Devilbiss et al., "The exosome complex establishes a barricade to erythroid maturation," Blood, vol. 124, no. 14, pp. 2285-2297, 2014.

[54] F. Collino, M. C. Deregibus, S. Bruno et al., "Microvesicles derived from adult human bone marrow and tissue specific mesenchymal stem cells shuttle selected pattern of miRNAs," PLoS ONE, vol. 5, no. 7, Article ID el1803, 2010.

[55] J. Zhang, J. Guan, X. Niu et al., "Exosomes released from human induced pluripotent stem cells-derived MSCs facilitate cutaneous wound healing by promoting collagen synthesis and angiogenesis," Journal of Translational Medicine, vol. 13, no. 1, p. 49, 2015. 
[56] A. Shabbir, A. Cox, L. Rodriguez-Menocal, M. Salgado, and E. V. Badiavas, "Mesenchymal stem cell exosomes induce proliferation and migration of normal and chronic wound fibroblasts, and enhance angiogenesis in vitro," Stem Cells and Development, vol. 24, no. 14, pp. 1635-1647, 2015.

[57] J. M. Aliotta, M. Pereira, M. Li et al., "Stable cell fate changes in marrow cells induced by lung-derived microvesicles," Journal of Extracellular Vesicles, vol. 1, 2012.

[58] P. J. Quesenberry, M. S. Dooner, and J. M. Aliotta, "Stem cell plasticity revisited: the continuum marrow model and phenotypic changes mediated by microvesicles," Experimental Hematology, vol. 38, no. 7, pp. 581-592, 2010.

[59] K. Takahashi and S. Yamanaka, "Induction of pluripotent stem cells from mouse embryonic and adult fibroblast cultures by defined factors," Cell, vol. 126, no. 4, pp. 663-676, 2006.

[60] Y. Tay, J. Zhang, A. M. Thomson, B. Lim, and I. Rigoutsos, "MicroRNAs to Nanog, Oct4 and Sox 2 coding regions modulate embryonic stem cell differentiation," Nature, vol. 455, no. 7216, pp. 1124-1128, 2008.

[61] N. Xu, T. Papagiannakopoulos, G. Pan, J. A. Thomson, and K. S. Kosik, "microRNA-145 regulates OCT4, SOX2, and KLF4 and represses pluripotency in human embryonic stem cells," Cell, vol. 137, no. 4, pp. 647-658, 2009.

[62] D. Katsman, E. J. Stackpole, D. R. Domin, and D. B. Farber, "Embryonic stem cell-derived microvesicles induce gene expression changes in Muller cells of the retina," PLOS ONE, vol. 7, no. 11, Article ID e50417, 2012.

[63] V. B. Cismasiu and L. M. Popescu, "Telocytes transfer extracellular vesicles loaded with microRNAs to stem cells," Journal of Cellular and Molecular Medicine, vol. 19, no. 2, pp. 351-358, 2015.

[64] W. Koh, C. T. Sheng, B. Tan et al., "Analysis of deep sequencing microRNA expression profile from human embryonic stem cells derived mesenchymal stem cells reveals possible role of let7 microRNA family in downstream targeting of hepatic nuclear factor 4 alpha," BMC Genomics, vol. 11, supplement 1, article S6, 2010.

[65] T. Oskarsson, E. Batlle, and J. Massagué, "Metastatic stem cells: sources, niches, and vital pathways," Cell Stem Cell, vol. 14, no. 3, pp. 306-321, 2014.

[66] A. M. Roccaro, A. Sacco, P. Maiso et al., "BM mesenchymal stromal cell-derived exosomes facilitate multiple myeloma progression," Journal of Clinical Investigation, vol. 123, no. 4, pp. 1542-1555, 2013.

[67] A. Eirin, S. M. Riester, X.-Y. Zhu et al., "microRNA and mRNA cargo of extracellular vesicles from porcine adipose tissuederived mesenchymal stem cells," Gene, vol. 551, no. 1, pp. 55-64, 2014.

[68] W. Zhu, L. Huang, Y. Li et al., "Exosomes derived from human bone marrow mesenchymal stem cells promote tumor growth in vivo," Cancer Letters, vol. 315, no. 1, pp. 28-37, 2012.

[69] K. C. Vallabhaneni, P. Penfornis, S. Dhule et al., "Extracellular vesicles from bone marrow mesenchymal stem/stromal cells transport tumor regulatory microRNA, proteins, and metabolites," Oncotarget, vol. 6, no. 7, pp. 4953-4967, 2015.

[70] M. Wang, C. Zhao, H. Shi et al., "Deregulated microRNAs in gastric cancer tissue-derived mesenchymal stem cells: novel biomarkers and a mechanism for gastric cancer," British Journal of Cancer, vol. 110, no. 5, pp. 1199-1210, 2014.

[71] R. S. Lindoso, F. Collino, and G. Camussi, "Extracellular vesicles derived from renal cancer stem cells induce a pro-tumorigenic phenotype in mesenchymal stromal cells," Oncotarget, vol. 6, no. 10, pp. 7959-7969, 2015.
[72] S. Bruno, F. Collino, M. C. Deregibus, C. Grange, C. Tetta, and G. Camussi, "Microvesicles derived from human bone marrow mesenchymal stem cells inhibit tumor growth," Stem Cells and Development, vol. 22, no. 5, pp. 758-771, 2013.

[73] M. Ono, N. Kosaka, N. Tominaga et al., "Exosomes from bone marrow mesenchymal stem cells contain a microRNA that promotes dormancy in metastatic breast cancer cells," Science Signaling, vol. 7, no. 332, p. ra63, 2014.

[74] J.-K. Lee, S.-R. Park, B.-K. Jung et al., "Exosomes derived from mesenchymal stem cells suppress angiogenesis by downregulating VEGF expression in breast cancer cells," PLoS ONE, vol. 8, no. 12, Article ID e84256, 2013.

[75] S. Wu, G.-Q. Ju, T. Du, Y.-J. Zhu, and G.-H. Liu, "Microvesicles derived from human umbilical cord Wharton's jelly mesenchymal stem cells attenuate bladder tumor cell growth in vitro and in vivo," PLoS ONE, vol. 8, no. 4, Article ID e61366, 2013.

[76] V. Fonsato, F. Collino, M. B. Herrera et al., "Human liver stem cell-derived microvesicles inhibit hepatoma growth in SCID mice by delivering antitumor microRNAs," Stem Cells, vol. 30, no. 9, pp. 1985-1998, 2012.

[77] S. Bruno, F. Collino, A. Iavello, and G. Camussi, "Effects of mesenchymal stromal cell-derived extracellular vesicles on tumor growth," Frontiers in Immunology, vol. 5, article 382, 2014.

[78] E. Bourkoula, D. Mangoni, T. Ius et al., "Glioma-associated stem cells: a novel class of tumor-supporting cells able to predict prognosis of human low-grade gliomas," Stem Cells, vol. 32, no. 5, pp. 1239-1253, 2014.

[79] J. L. Munoz, S. A. Bliss, S. J. Greco, S. H. Ramkissoon, K. L. Ligon, and P. Rameshwar, "Delivery of functional anti-miR-9 by mesenchymal stem cell-derived exosomes to glioblastoma multiforme cells conferred chemosensitivity," Molecular TherapyNucleic Acids, vol. 2, article e126, 2013.

[80] A. Bronisz, Y. Wang, M. O. Nowicki et al., "Extracellular vesicles modulate the glioblastoma microenvironment via a tumor suppression signaling network directed by miR-1," Cancer Research, vol. 74, no. 3, pp. 738-750, 2014.

[81] I. Nakano, D. Garnier, M. Minata, and J. Rak, "Extracellular vesicles in the biology of brain tumour stem cells-implications for inter-cellular communication, therapy and biomarker development," Seminars in Cell \& Developmental Biology, vol. 40, pp. 17-26, 2015.

[82] M. Katakowski, B. Buller, X. Zheng et al., "Exosomes from marrow stromal cells expressing miR-146b inhibit glioma growth," Cancer Letters, vol. 335, no. 1, pp. 201-204, 2013.

[83] L. Pascucci, V. Coccè, A. Bonomi et al., "Paclitaxel is incorporated by mesenchymal stromal cells and released in exosomes that inhibit in vitro tumor growth: a new approach for drug delivery," Journal of Controlled Release, vol. 192, pp. 262-270, 2014.

[84] V. Plaks, N. Kong, and Z. Werb, "The cancer stem cell niche: how essential is the niche in regulating stemness of tumor cells?" Cell Stem Cell, vol. 16, no. 3, pp. 225-238, 2015.

[85] R. Ghasemi, A. Grassadonia, N. Tinari et al., "Tumor-derived microvesicles: the metastasomes," Medical Hypotheses, vol. 80, no. 1, pp. 75-82, 2013.

[86] M. H. Barcellos-Hoff, D. Lyden, and T. C. Wang, "The evolution of the cancer niche during multistage carcinogenesis," Nature Reviews Cancer, vol. 13, no. 7, pp. 511-518, 2013.

[87] H. Haga, I. K. Yan, K. Takahashi, J. Wood, A. Zubair, and T. Patel, "Tumour cell-derived extracellular vesicles interact with mesenchymal stem cells to modulate the microenvironment and 
enhance cholangiocarcinoma growth," Journal of Extracellular Vesicles, vol. 4, Article ID 24900, 2015.

[88] J. A. Cho, H. Park, E. H. Lim et al., "Exosomes from ovarian cancer cells induce adipose tissue-derived mesenchymal stem cells to acquire the physical and functional characteristics of tumorsupporting myofibroblasts," Gynecologic Oncology, vol. 123, no. 2, pp. 379-386, 2011.

[89] R. Chowdhury, J. P. Webber, M. Gurney, M. D. Mason, Z. Tabi, and A. Clayton, "Cancer exosomes trigger mesenchymal stem cell differentiation into pro-angiogenic and pro-invasive myofibroblasts," Oncotarget, vol. 6, no. 2, pp. 715-731, 2015.

[90] Z. Y. A. Elmageed, Y. Yang, R. Thomas et al., "Neoplastic reprogramming of patient-derived adipose stem cells by prostate cancer cell-associated exosomes," Stem Cells, vol. 32, no. 4, pp. 983-997, 2014.

[91] J. Gu, H. Qian, L. Shen et al., "Gastric cancer exosomes trigger differentiation of umbilical cord derived mesenchymal stem cells to carcinoma-associated fibroblasts through TGF- $\beta /$ smad pathway," PLoS ONE, vol. 7, no. 12, Article ID e52465, 2012.

[92] M. Shimoda, S. Principe, H. W. Jackson et al., "Loss of the Timp gene family is sufficient for the acquisition of the CAF-like cell state," Nature Cell Biology, vol. 16, no. 9, pp. 889-901, 2014.

[93] Y. Hu, C. Yan, L. Mu et al., "Fibroblast-derived exosomes contribute to chemoresistance through priming cancer stem cells in colorectal cancer," PLoS ONE, vol. 10, no. 5, Article ID e0125625, 2015.

[94] M. Quante, S. P. Tu, H. Tomita et al., "Bone marrow-derived myofibroblasts contribute to the mesenchymal stem cell niche and promote tumor growth," Cancer Cell, vol. 19, no. 2, pp. 257272, 2011.

[95] A. Yuan, E. L. Farber, A. L. Rapoport et al., "Transfer of microRNAs by embryonic stem cell microvesicles," PLoS ONE, vol. 4, no. 3, Article ID e4722, 2009.

[96] R. Koch, M. Demant, T. Aung et al., "Populational equilibrium through exosome-mediated Wnt signaling in tumor progression of diffuse large B-cell lymphoma," Blood, vol. 123, no. 14, pp. 2189-2198, 2014.

[97] M. J. Rahman, D. Regn, R. Bashratyan, and Y. D. Dai, “Exosomes released by islet-derived mesenchymal stem cells trigger autoimmune responses in NOD mice," Diabetes, vol. 63, no. 3 , pp. 1008-1020, 2014.

[98] F. T. Borges, S. A. Melo, B. C. Özdemir et al., "TGF-betalcontaining exosomes from injured epithelial cells activate fibroblasts to initiate tissue regenerative responses and fibrosis," Journal of the American Society of Nephrology, vol. 24, no. 3, pp. 385-392, 2013.

[99] Y.-G. Zhu, X.-M. Feng, J. Abbott et al., "Human mesenchymal stem cell microvesicles for treatment of Escherichia coli endotoxin-induced acute lung injury in mice," Stem Cells, vol. 32, no. 1, pp. 116-125, 2014.

[100] S. Tomasoni, L. Longaretti, C. Rota et al., "Transfer of growth factor receptor mRNA via exosomes unravels the regenerative effect of mesenchymal stem cells," Stem Cells and Development, vol. 22, no. 5, pp. 772-780, 2013.

[101] J. Aoki, K. Ohashi, M. Mitsuhashi et al., "Posttransplantation bone marrow assessment by quantifying hematopoietic cellderived mRNAs in plasma exosomes/microvesicles," Clinical Chemistry, vol. 60, no. 4, pp. 675-682, 2014.

[102] S. Sahoo, E. Klychko, T. Thorne et al., "Exosomes from human $\mathrm{CD} 34^{+}$stem cells mediate their proangiogenic paracrine activity," Circulation Research, vol. 109, no. 7, pp. 724-728, 2011.
[103] T. Li, Y. Yan, B. Wang et al., "Exosomes derived from human umbilical cord mesenchymal stem cells alleviate liver fibrosis," Stem Cells and Development, vol. 22, no. 6, pp. 845-854, 2013.

[104] S.-S. Lin, B. Zhu, Z.-K. Guo et al., "Bone marrow mesenchymal stem cell-derived microvesicles protect rat pheochromocytoma PC12 cells from glutamate-induced injury via a PI3K/Akt dependent pathway," Neurochemical Research, vol. 39, no. 5, pp. 922-931, 2014.

[105] T. Katsuda, R. Tsuchiya, N. Kosaka et al., "Human adipose tissue-derived mesenchymal stem cells secrete functional neprilysin-bound exosomes," Scientific Reports, vol. 3, article 1197, 2013.

[106] J.-F. Xu, G.-H. Yang, X.-H. Pan et al., "Altered microRNA expression profile in exosomes during osteogenic differentiation of human bone marrow-derived mesenchymal stem cells," PLoS ONE, vol. 9, no. 12, Article ID el14627, 2014.

[107] H. Xin, Y. Li, B. Buller et al., "Exosome-mediated transfer of miR-133b from multipotent mesenchymal stromal cells to neural cells contributes to neurite outgrowth," Stem Cells, vol. 30, no. 7, pp. 1556-1564, 2012.

[108] R. Gernapudi, Y. Yao, Y. Zhang et al., "Targeting exosomes from preadipocytes inhibits preadipocyte to cancer stem cell signaling in early-stage breast cancer," Breast Cancer Research and Treatment, vol. 150, no. 3, pp. 685-695, 2015.

[109] Y. Feng, W. Huang, M. Wani, X. Yu, and M. Ashraf, "Ischemic preconditioning potentiates the protective effect of stem cells through secretion of exosomes by targeting Mecp2 via miR-22," PLoS ONE, vol. 9, no. 2, Article ID e88685, 2014.

[110] B. Yu, M. Gong, Y. Wang et al., "Cardiomyocyte protection by GATA-4 gene engineered mesenchymal stem cells is partially mediated by translocation of miR-221 in microvesicles," PLoS ONE, vol. 8, no. 8, Article ID e73304, 2013.

[111] L. Barile, V. Lionetti, E. Cervio et al., "Extracellular vesicles from human cardiac progenitor cells inhibit cardiomyocyte apoptosis and improve cardiac function after myocardial infarction," Cardiovascular Research, vol. 103, no. 4, pp. 530-541, 2014.

[112] M. Khan, E. Nickoloff, T. Abramova et al., "Embryonic stem cell-derived exosomes promote endogenous repair mechanisms and enhance cardiac function following myocardial infarction," Circulation Research, vol. 117, no. 1, pp. 52-64, 2015.

[113] J. Wang, S. Chen, X. Ma et al., "Effects of endothelial progenitor cell-derived microvesicles on hypoxia/reoxygenation-induced endothelial dysfunction and apoptosis," Oxidative Medicine and Cellular Longevity, vol. 2013, Article ID 572729, 9 pages, 2013.

[114] X.-Y. Bi, S. Huang, J.-L. Chen, F. Wang, Y. Wang, and Z.-K. Guo, "Exploration of conditions for releasing microvesicle from human bone marrow mesenchymal stem cells," Zhongguo Shi Yan Xue Ye Xue Za Zhi, vol. 22, no. 2, pp. 491-495, 2014.

[115] T. Lopatina, S. Bruno, C. Tetta, N. Kalinina, M. Porta, and G. Camussi, "Platelet-derived growth factor regulates the secretion of extracellular vesicles by adipose mesenchymal stem cells and enhances their angiogenic potential," Cell Communication and Signaling, vol. 12, article 26, 2014.

[116] L. Pascucci, G. Alessandri, C. Dall'Aglio et al., "Membrane vesicles mediate pro-angiogenic activity of equine adiposederived mesenchymal stromal cells," Veterinary Journal, vol. 202, no. 2, pp. 361-366, 2014.

[117] L. Zitvogel, A. Regnault, A. Lozier et al., "Eradication of established murine tumors using a novel cell-free vaccine: dendritic cell-derived exosomes," Nature Medicine, vol. 4, no. 5, pp. 594-600, 1998. 
[118] N. M. Mahaweni, M. E. Kaijen-Lambers, J. Dekkers, J. G. Aerts, and J. P. Hegmans, "Tumour-derived exosomes as antigen delivery carriers in dendritic cell-based immunotherapy for malignant mesothelioma," Journal of Extracellular Vesicles, vol. 2, 2013.

[119] G. Raposo, H. W. Nijman, W. Stoorvogel et al., "B lymphocytes secrete antigen-presenting vesicles," Journal of Experimental Medicine, vol. 183, no. 3, pp. 1161-1172, 1996.

[120] B. Zhang, Y. Yin, R. C. Lai, S. S. Tan, A. B. H. Choo, and S. K. Lim, "Mesenchymal stem cells secrete immunologically active exosomes," Stem Cells and Development, vol. 23, no. 11, pp. 12331244, 2014.

[121] R. Blazquez, F. M. Sanchez-Margallo, O. de la Rosa et al., "Immunomodulatory potential of human adipose mesenchymal stem cells derived exosomes on in vitro stimulated T cells," Frontiers in Immunology, vol. 5, article 556, 2014.

[122] K. Ekström, O. Omar, C. Granéli, X. Wang, F. Vazirisani, and P. Thomsen, "Monocyte exosomes stimulate the osteogenic gene expression of mesenchymal stem cells," PLoS ONE, vol. 8, no. 9, Article ID e75227, 2013.

[123] C. S. Hong, L. Muller, M. Boyiadzis, and T. L. Whiteside, "Isolation and characterization of CD34+ blast-derived exosomes in acute myeloid leukemia," PLoS ONE, vol. 9, no. 8, Article ID e103310, 2014.

[124] S. Amarnath, J. E. Foley, D. E. Farthing et al., "Bone marrowderived mesenchymal stromal cells harness purinergenic signaling to tolerize human Th1 cells in vivo," Stem Cells, vol. 33, no. 4, pp. 1200-1212, 2015.

[125] L. Kordelas, V. Rebmann, A.-K. Ludwig et al., "MSC-derived exosomes: a novel tool to treat therapy-refractory graft-versushost disease," Leukemia, vol. 28, no. 4, pp. 970-973, 2014.

[126] B. Zhang, Y. Yin, R. C. Lai, and S. K. Lim, "Immunotherapeutic potential of extracellular vesicles," Frontiers in Immunology, vol. 5, article 518, 2014.

[127] E. I. Buzas, B. György, G. Nagy, A. Falus, and S. Gay, "Emerging role of extracellular vesicles in inflammatory diseases," Nature Reviews Rheumatology, vol. 10, no. 6, pp. 356-364, 2014.

[128] C. Lee, S. A. Mitsialis, M. Aslam et al., "Exosomes mediate the cytoprotective action of mesenchymal stromal cells on hypoxiainduced pulmonary hypertension," Circulation, vol. 126, no. 22, pp. 2601-2611, 2012.

[129] F. Arslan, R. C. Lai, M. B. Smeets et al., "Mesenchymal stem cellderived exosomes increase ATP levels, decrease oxidative stress and activate PI3K/Akt pathway to enhance myocardial viability and prevent adverse remodeling after myocardial ischemia/ reperfusion injury," Stem Cell Research, vol. 10, no. 3, pp. 301312, 2013.

[130] K. R. Vrijsen, J. P. G. Sluijter, M. W. L. Schuchardt et al., "Cardiomyocyte progenitor cell-derived exosomes stimulate migration of endothelial cells," Journal of Cellular and Molecular Medicine, vol. 14, no. 5, pp. 1064-1070, 2010.

[131] S. Bian, L. Zhang, L. Duan, X. Wang, Y. Min, and H. Yu, "Extracellular vesicles derived from human bone marrow mesenchymal stem cells promote angiogenesis in a rat myocardial infarction model," Journal of Molecular Medicine, vol. 92, no. 4, pp. 387-397, 2014.

[132] K. Kang, R. Ma, W. Cai et al., "Exosomes secreted from CXCR4 overexpressing mesenchymal stem cells promote cardioprotection via Akt signaling pathway following myocardial infarction," Stem Cells International, vol. 2015, Article ID 659890, 14 pages, 2015.
[133] Z. Giricz, Z. V. Varga, T. Baranyai et al., "Cardioprotection by remote ischemic preconditioning of the rat heart is mediated by extracellular vesicles," Journal of Molecular and Cellular Cardiology, vol. 68, pp. 75-78, 2014.

[134] Y. Feng, W. Huang, W. Meng et al., "Heat shock improves Sca-1+ stem cell survival and directs ischemic cardiomyocytes toward a prosurvival phenotype via exosomal transfer: a critical role for HSF1/miR-34a/HSP70 pathway," Stem Cells, vol. 32, no. 2, pp. 462-472, 2014.

[135] B. Yu, H. W. Kim, M. Gong et al., "Exosomes secreted from GATA-4 overexpressing mesenchymal stem cells serve as a reservoir of anti-apoptotic microRNAs for cardioprotection," International Journal of Cardiology, vol. 182, pp. 349-360, 2015.

[136] M. Choi, T. Ban, and T. Rhim, "Therapeutic use of stem cell transplantation for cell replacement or cytoprotective effect of microvesicle released from mesenchymal stem cell," Molecules and Cells, vol. 37, no. 2, pp. 133-139, 2014.

[137] B. D. Humphreys, M. T. Valerius, A. Kobayashi et al., "Intrinsic epithelial cells repair the kidney after injury," Cell Stem Cell, vol. 2, no. 3, pp. 284-291, 2008.

[138] B. Bi, R. Schmitt, M. Israilova, H. Nishio, and L. G. Cantley, "Stromal cells protect against acute tubular injury via an endocrine effect," Journal of the American Society of Nephrology, vol. 18, no. 9, pp. 2486-2496, 2007.

[139] S. Bruno, C. Grange, M. C. Deregibus et al., "Mesenchymal stem cell-derived microvesicles protect against acute tubular injury," Journal of the American Society of Nephrology, vol. 20, no. 5, pp. 1053-1067, 2009.

[140] Y. Zhou, H. Xu, W. Xu et al., "Exosomes released by human umbilical cord mesenchymal stem cells protect against cisplatin-induced renal oxidative stress and apoptosis in vivo and in vitro," Stem Cell Research and Therapy, vol. 4, no. 2, article 34, 2013.

[141] S. Gatti, S. Bruno, M. C. Deregibus et al., "Microvesicles derived from human adult mesenchymal stem cells protect against ischaemia-reperfusion-induced acute and chronic kidney injury," Nephrology Dialysis Transplantation, vol. 26, no. 5, pp. 1474-1483, 2011.

[142] S. Bruno, C. Grange, F. Collino et al., "Microvesicles derived from mesenchymal stem cells enhance survival in a lethal model of acute kidney injury," PLoS ONE, vol. 7, no. 3, Article ID e33115, 2012.

[143] R. S. Lindoso, F. Collino, S. Bruno et al., "Extracellular vesicles released from mesenchymal stromal cells modulate miRNA in renal tubular cells and inhibit ATP depletion injury," Stem Cells and Development, vol. 23, no. 15, pp. 1809-1819, 2014.

[144] F. Collino, S. Bruno, D. Incarnato et al., "AKI recovery induced by mesenchymal stromal cell-derived extracellular vesicles carrying MicroRNAs," Journal of the American Society of Nephrology, 2015.

[145] J. He, Y. Wang, S. Sun et al., "Bone marrow stem cells-derived microvesicles protect against renal injury in the mouse remnant kidney model," Nephrology, vol. 17, no. 5, pp. 493-500, 2012.

[146] V. Cantaluppi, D. Medica, C. Mannari et al., "Endothelial progenitor cell-derived extracellular vesicles protect from complement-mediated mesangial injury in experimental antiThyl.1 glomerulonephritis," Nephrology Dialysis Transplantation, vol. 30, no. 3, pp. 410-422, 2015.

[147] M. Sanchez, S. Bruno, C. Grange et al., "Human liver stem cells and derived extracellular vesicles improve recovery in a murine model of acute kidney injury," Stem Cell Research \& Therapy, vol. 5, no. 6, article 124, 2014. 
[148] J. He, Y. Wang, X. Lu et al., "Micro-vesicles derived from bone marrow stem cells protect the kidney both in vivo and in vitro by microRNA-dependent repairing," Nephrology, vol. 20, no. 9, pp. 591-600, 2015.

[149] J. M. Aliotta, F. M. Sanchez-Guijo, G. J. Dooner et al., "Alteration of marrow cell gene expression, protein production, and engraftment into lung by lung-derived microvesicles: a novel mechanism for phenotype modulation," Stem Cells, vol. 25, no. 9, pp. 2245-2256, 2007.

[150] L. Li, S. Jin, and Y. Zhang, "Ischemic preconditioning potentiates the protective effect of mesenchymal stem cells on endotoxin-induced acute lung injury in mice through secretion of exosome," International Journal of Clinical and Experimental Medicine, vol. 8, no. 3, pp. 3825-3832, 2015.

[151] M. B. Herrera, V. Fonsato, S. Gatti et al., "Human liver stem cellderived microvesicles accelerate hepatic regeneration in hepatectomized rats," Journal of Cellular and Molecular Medicine, vol. 14, no. 6B, pp. 1605-1618, 2010.

[152] C. Y. Tan, R. C. Lai, W. Wong, Y. Y. Dan, S.-K. Lim, and H. K. Ho, "Mesenchymal stem cell-derived exosomes promote hepatic regeneration in drug-induced liver injury models," Stem Cell Research and Therapy, vol. 5, no. 3, article 76, 2014.

[153] A. Raisi, S. Azizi, N. Delirezh, B. Heshmatian, A. A. Farshid, and K. Amini, "The mesenchymal stem cell-derived microvesicles enhance sciatic nerve regeneration in rat: a novel approach in peripheral nerve cell therapy," Journal of Trauma and Acute Care Surgery, vol. 76, no. 4, pp. 991-997, 2014.

[154] D. M. Feliciano, S. Zhang, C. M. Nasrallah, S. N. Lisgo, and A. Bordey, "Embryonic cerebrospinal fluid nanovesicles carry evolutionarily conserved molecules and promote neural stem cell amplification," PLoS ONE, vol. 9, no. 2, Article ID e88810, 2014.

[155] X. Zhuang, X. Xiang, W. Grizzle et al., "Treatment of brain inflammatory diseases by delivering exosome encapsulated anti-inflammatory drugs from the nasal region to the brain," Molecular Therapy, vol. 19, no. 10, pp. 1769-1779, 2011.

[156] Y. Zhang, M. Chopp, Y. Meng et al., "Effect of exosomes derived from multipluripotent mesenchymal stromal cells on functional recovery and neurovascular plasticity in rats after traumatic brain injury," Journal of Neurosurgery, vol. 122, no. 4, pp. 856$867,2015$.

[157] P. J. Quesenberry, G. Dooner, M. Dooner, and M. Abedi, "Developmental biology: ignoratio elenchi: red herrings in stem cell research," Science, vol. 308, no. 5725, pp. 1121-1122, 2005.

[158] R. J. Simpson, H. Kalra, and S. Mathivanan, "ExoCarta as a resource for exosomal research," Journal of Extracellular Vesicles, vol. 1, Article ID 18374, 2012.

[159] D. Kumar, D. Gupta, S. Shankar, and R. K. Srivastava, "Biomolecular characterization of exosomes released from cancer stem cells: possible implications for biomarker and treatment of cancer," Oncotarget, vol. 6, no. 5, pp. 3280-3291, 2015. 

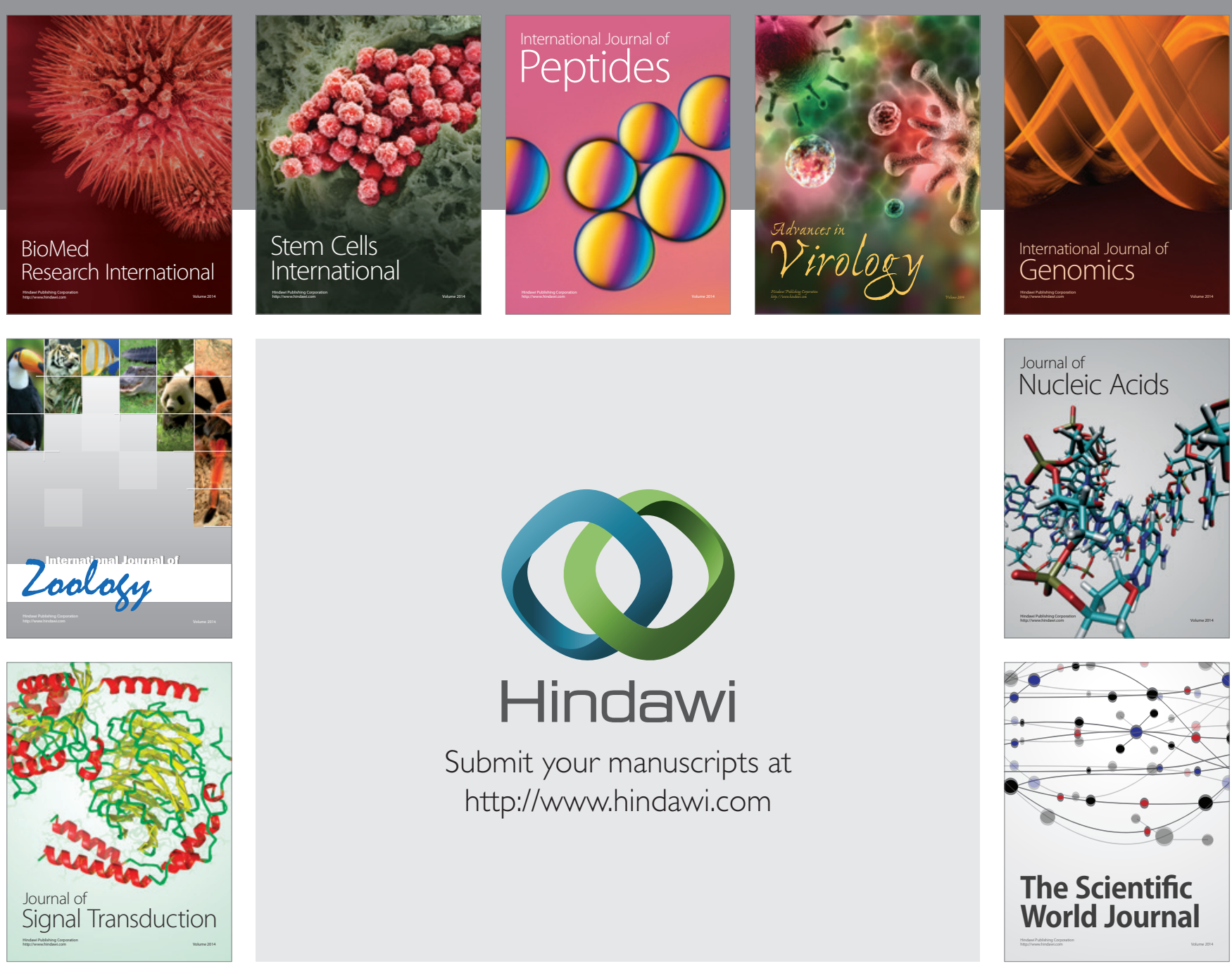

Submit your manuscripts at

http://www.hindawi.com
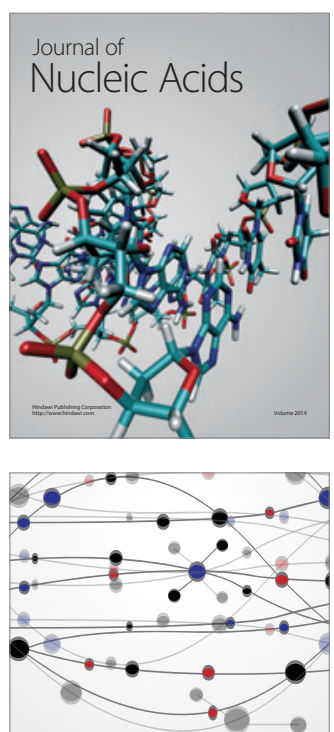

The Scientific World Journal
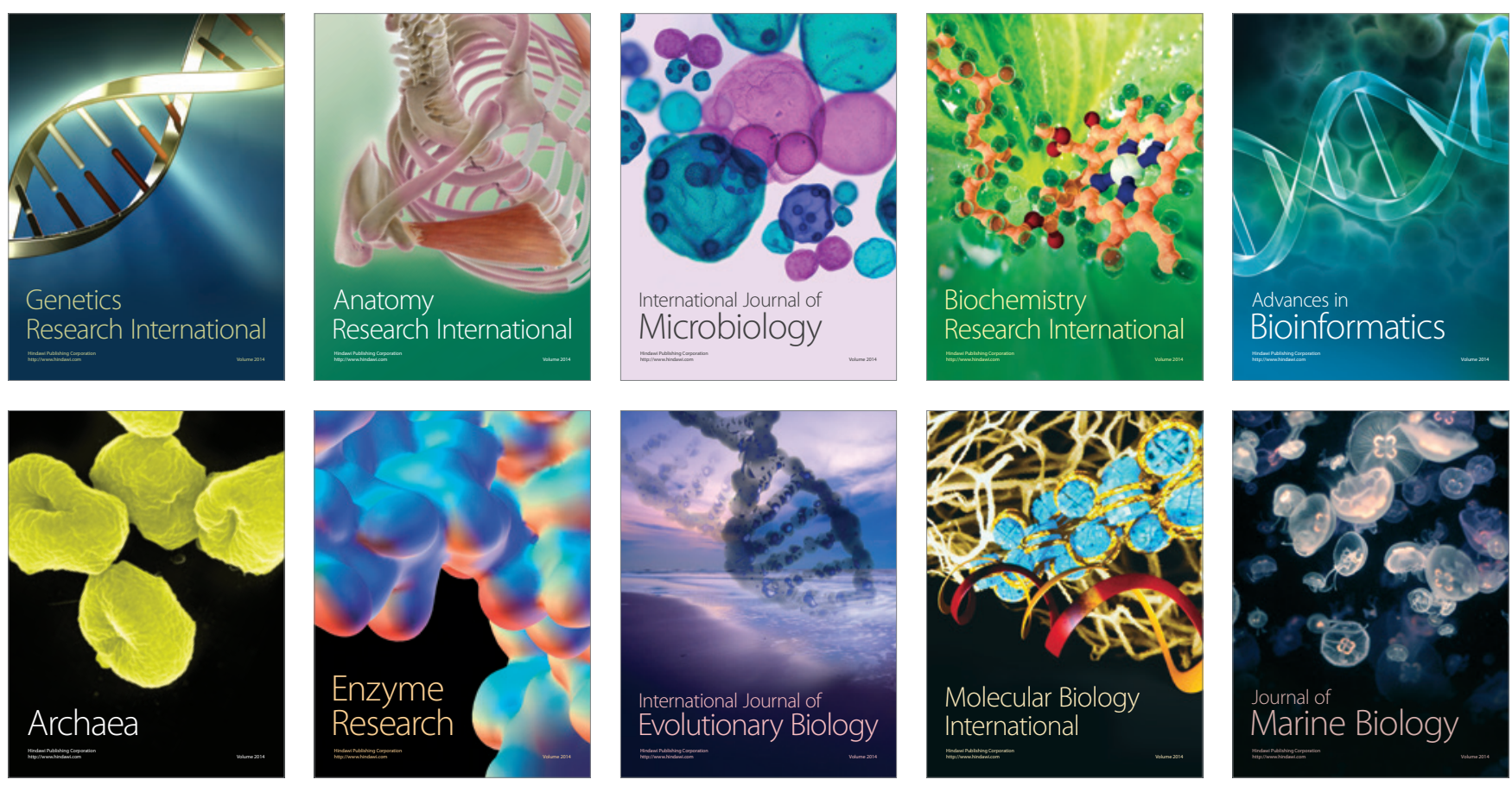